\title{
A Cuttable Wireless Power Transfer Sheet
}

\author{
RYO TAKAHASHI, The University of Tokyo, Japan \\ TAKUYA SASATANI, The University of Tokyo, Japan and JSPS Research Fellow, Japan \\ FUMINORI OKUYA, The University of Tokyo, Japan \\ YOSHIAKI NARUSUE, The University of Tokyo, Japan \\ YOSHIHIRO KAWAHARA, The University of Tokyo, Japan
}

\begin{abstract}
We propose a cuttable wireless power transfer sheet which allows users to modify its size and shape. This intuitive manipulation allows users to easily add wireless power transmission capabilities to everyday objects. The properties of the sheet such as thinness, flexibility, and lightness make our sheet highly compatible with various configurations. We contribute a set of technical principles for the design of circuitry, which integrates $\mathrm{H}$-tree wiring and time division power supply techniques. $\mathrm{H}$-tree wiring allows the sheet to remain functional even when cut from the outside of the sheet, whereas time division power supply avoids the reduction in power transfer efficiency caused by the magnetic interference between adjacent transmitter coils. Through the evaluations, we found that our time division power supply scheme mitigates the degradation of power transfer efficiency and successfully improves the average efficiency. Furthermore, we present four applications which integrates our sheet into daily objects: wireless charging furniture, bag, jacket, and craft; these applications confirmed the feasibility of our prototype.
\end{abstract}

CCS Concepts: • Human-centered computing $\rightarrow$ Ubiquitous and mobile computing;

Additional Key Words and Phrases: Wireless power transfer sheet, cuttable electronics, thin film coil array

ACM Reference Format:

Ryo Takahashi, Takuya Sasatani, Fuminori Okuya, Yoshiaki Narusue, and Yoshihiro Kawahara. 2018. A Cuttable Wireless Power Transfer Sheet. Proc. ACM Interact. Mob. Wearable Ubiquitous Technol. 2, 4, Article 190 (December 2018), 25 pages. https://doi.org/10.1145/3287068

\section{INTRODUCTION}

Wireless Power Transfer (WPT) using designated charging cradles has reached the stage of commercialization in the past few decades $[1,2]$. Although this provides some advantages over wired power supply such as being waterproof and having no mechanical contacts, it still requires a dedicated transmitter (TX) and perfectly aligned placement of a receiver (RX). To achieve hassle-free integration of charging functions into daily objects, it is necessary to offer a "novice-friendly" design procedure for integrating WPT capabilities with users' convenience. A straightforward approach toward this concept is to form WPT surfaces by arranging TX coils according to the shape of target surfaces $[1,17,25,26,38]$. However, the limitation of this approach is that this design procedure

Authors' addresses: Ryo Takahashi, The University of Tokyo, 7-3-1 Hongo, Bunkyo-ku, Tokyo, 113-8656, Japan, takahashi@akg.t.u-tokyo.ac.jp; Takuya Sasatani, The University of Tokyo, 7-3-1 Hongo, Bunkyo-ku, Tokyo, 113-8656, Japan, JSPS Research Fellow, Tokyo, Japan, sasatani@ akg.t.u-tokyo.ac.jp; Fuminori Okuya, The University of Tokyo, 7-3-1 Hongo, Bunkyo-ku, Tokyo, 113-8656, Japan, okuya23@akg.t.u-tokyo.ac.jp; Yoshiaki Narusue, The University of Tokyo, 7-3-1 Hongo, Bunkyo-ku, Tokyo, 113-8656, Japan, narusue@mlab.t.u-tokyo.ac.jp; Yoshihiro Kawahara, The University of Tokyo, 7-3-1 Hongo, Bunkyo-ku, Tokyo, 113-8656, Japan, kawahara@akg.t.u-tokyo.ac.jp.

Permission to make digital or hard copies of all or part of this work for personal or classroom use is granted without fee provided that copies are not made or distributed for profit or commercial advantage and that copies bear this notice and the full citation on the first page. Copyrights for components of this work owned by others than ACM must be honored. Abstracting with credit is permitted. To copy otherwise, or republish, to post on servers or to redistribute to lists, requires prior specific permission and/or a fee. Request permissions from permissions@acm.org.

(c) 2018 Association for Computing Machinery.

2474-9567/2018/12-ART190 \$15.00

https://doi.org/10.1145/3287068

Proc. ACM Interact. Mob. Wearable Ubiquitous Technol., Vol. 2, No. 4, Article 190. Publication date: December 2018. 

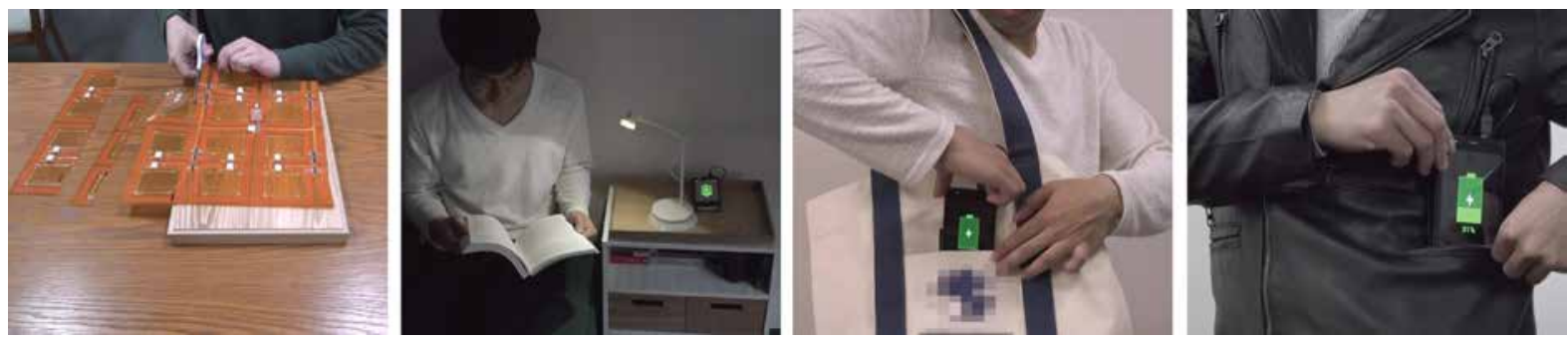

Fig. 1. Our cuttable WPT sheet allows the fabrication of WPT surfaces through a simple physical "cut-and-paste" process. We introduce three application examples: wireless charging shelf, bag, and jacket.

necessitates tremendous effort, knowledge, and time. This is not just a problem of designing the coil array topology so that it fits in the intended shape, but also involves a complex wiring process; this process brings about stricter topological constraints as well as compensation of the non-ideal effects that occur due to high-frequency operation [38]. Furthermore, when TX coils form a dense array, the magnetic interference between adjacent TX coils is non-negligible; this causes a reduction in power transfer efficiency, which is another challenge that must be addressed $[6,24]$.

To push the boundaries of ubiquitous WPT surfaces (i.e., power delivery to devices placed on everyday surfaces), we propose a cuttable WPT sheet that allows the fabrication of WPT surfaces through a simple physical "cutand-paste" process. Our idea began by observing our surroundings - we noticed that the customizability and simplicity of traditional cuttable resources such as wood, cloth, and paper have accelerated the fabrication of custom objects. Carpenters cut out standardized wooden boards to build furniture such as desks, chairs, and shelves, whereas tailors cut ready-made textiles to make clothes fit different sizes of the customer. Cardboard and plywood are frequently cut by designers to make physical prototypes and wrapping materials are cut to cover objects. Thus, we believe that by using our analogy concept - "cuttable WPT sheet" -, anyone (e.g., researchers, hobbyists, craft-men, and end users) should be able to quickly transform various daily surfaces into ubiquitous WPT surfaces; some example applications are shown in Fig. 1. Some example scenarios we envision are: (a) manufacturers offer wooden boards with our WPT sheet embedded, and carpenters and DIYers cut these WPT boards to build wireless charging enabled furniture, and (b) the fashion industry embeds our WPT sheet in clothing/bags and enable devices to be charged as they are simply placed in pockets.

To provide a proof of concept, we present the first prototype of a cuttable WPT sheet. Our design combines $\mathrm{H}$-tree wiring and time division power supply; $\mathrm{H}$-tree wiring makes our sheet capable of being cut, whereas time division power supply prevents magnetic interference between adjacent TX coils. We implemented a functional prototype of the proposed sheet using commercial off-the-shelf (COTS) components and a flexible PCB; the size and weight of our prototype are $400 \mathrm{~mm} \times 400 \mathrm{~mm}$ and $512 \mathrm{~g} / \mathrm{m}^{2}$, respectively. Furthermore, we present a wide range of applications and conduct a series of technical evaluations, to show typical use-cases, capabilities, limitations of our design. The application scenarios we consider are 1) wireless charging furniture, 2) wireless charging bag, 3) wireless charging jacket, and 4) wireless charging craft.

The main contributions of this work can be summarized as follows:

1. Introduction and design guidelines for a cuttable WPT sheet

2. Implementation and evaluation of our prototype

3. Exploration of a range of applications

In the following sections, we will present the related works, functions of our sheet, implementation details, potential applications, technical evaluations, limitations, and future works. 


\section{BACKGROUND AND RELATED WORKS}

Our work is strongly related to the following research areas: cuttable electronics and 2-D power supply sheets. In this section, we first introduce cuttable electronics which our work is inspired by. Then, we review potential technologies for enabling our vision of ubiquitous WPT surfaces, which include ad-hoc contact DC power supply sheets and WPT sheets.

\subsection{Cuttable Electronics}

Our work is heavily inspired by prior research on cuttable electronics, which can be categorized into two groups: 1) modular and programmable 1-D sensor arrays [9, 11, 15, 49] and 2) cuttable 2-D sensor sheets [33]. The former can be separated and rejoined to make various shapes. In contrast, the latter can be customized into various shapes by cutting; our work aims to boost the latter trend of cuttable electronics by adding "power supply" capability, which is one of the most highly desired and fundamental primitives of electronics.

Wiring topology is a key factor in cuttable sensor sheets since they achieve ad-hoc customization by cutting. Olberding et al. proposed to connect touch sensors through the combination of tree and star topologies so that the remaining sensors do not lose connection to the central unit upon cutting [33]. These topologies are known to exist in biological system and computer network studies; since they draw wires from the center to the edges, they are robust against outer edge faults.

\subsection{Power Supply Sheets}

Power supply sheets are functional 2-D surfaces that provide power to the RXs placed on them. We categorize these researches into two groups: 1) ad-hoc contact DC power supply sheets [13, 37, 39, 42, 44, 46] and 2) WPT sheets $[17,25,26,30,35,38,45]$. Ad-hoc contact DC power supply sheets transmit power to RXs via electrodes which establish conductive contact. In contrast, WPT sheets transfer power via electromagnetic fields in a contactless manner [5]. Given that typical surfaces are coated or painted (e.g., wallpaper, desk, shelf, etc.), contact-less is a mandatory factor. Therefore, our study focuses on advancing the field of WPT sheets.

Various approaches towards WPT sheets have been introduced, ranging from inductive power transfer (IPT) [38, 43], magnetically coupling resonant wireless power transfer (MCR-WPT) [14, 17, 25, 26], capacitive power transfer (CPT) [8, 41], 2-D waveguide power transmission [30, 31], quasi-wireless power transfer (Quasi-WPT) [35, 45], and so on. In the following, we review previous WPT sheets in terms of wiring topology and system architectures.

IPT transfers energy via the magnetic coupling between TX/RX coils and can achieve high-efficiency power transfer over a short distance [12]. Qi standard can be extended to 2-D surfaces by composing a switchable TX coil array, in which each TX coil is connected to the power source and the central control unit activates the TXs closest to RXs [4]. While this connection can be easily applied to a few TX coils, assuring the space for wiring of tens of TX coils in this architecture is a challenge. In contrast, the TX coil array by Sekitani et al. employs matrix topology for selecting TX coils close to RXs [38,43]. The elegance of this approach is that the number of wires can be minimized [33], but the disconnection of a single line results in the irreversible dysfunction of many TX coils. Hence, this is not suitable for shape adaptation by cutting.

MCR-WPT is a variant of IPT which employs high-Q magnetically coupled TX/RX resonators instead of TX/RX coils and has the promise of high-efficiency power delivery even under loosely coupled conditions; consequently, misalignment tolerance can be improved [20]. Furthermore, it is known that by placing resonant coils next to the TX, the magnetic field can be "relayed" and as a result, the power transfer distance can be extended [40]. Two major types of MCR-WPT sheets have been presented in literature: 1) switchable TX coil array sheets [17, 25] and 2) relay coil array sheets [14, 26]. Mori et al. [25] and Jolani et al. [17] apply MCR-WPT to switchable TX coil arrays, but they are based on matrix topology similar to Sekitani et al. [38] and thus, are not suitable for shape adaptation by cutting, as described above. On the other hand, relay coil array sheets 
are good in that the relay coils do not have to be connected to each other; however, the relay coils have to be precisely controlled to direct energy as intended, and therefore a powerful central unit as well as a protocol are necessary $[14,26]$.

CPT is an approach that employs capacitive coupling and achieves high-efficiency over a short distance. The TX/RX of traditional CPT system consist of two pairs of metal plates [21]. While CPT systems are easy to fabricate, there is a typical problem in that the power transfer efficiency is easily degraded by 1) the misalignment of TX/RX plates and 2) the dielectric materials around the plates such as wood, cardboard, plastic, or plywood; this is critical since there are many everyday materials that work as dielectrics and they exist all over the real world. Although methods to improve misalignment tolerance are currently being investigated and have succeeded in specific configurations [8], detouring the effect of dielectrics still requires complex control mechanisms such as adaptive impedance tuning [41].

2-D waveguide power transmission transfers energy via non-radiative electromagnetic standing waves generated within a custom designed 2-D waveguide sheet. The TX stimulates the resonant modes of the sheet via an incident signal and generates the standing waves, whereas the RX couples with the standing waves and receives energy [30,31]. While this system enables large-area 2D waveguide power transmission at low cost due to its simple waveguide structure, the relatively low efficiency and the null points of power delivery are its non-negligible disadvantages [31]. Noda et al. showed that it is possible to avoid the generation of null points by selecting an appropriate standing wave pattern according to a single RX position [31]. Another critical drawback is that the characteristic of the modes (i.e., field pattern, frequency, etc.) are fully determined by sheet geometry; the modification of the shape requires the design of an entirely new driving system and thus, modification is not suitable.

Quasi-WPT is a recently presented method based on single-wire no-return power transmission [35, 45]. This method powers electronic devices over conductive surfaces using standing wave modes excited within a helical receiver $[35,45]$. The TX in this system can be fabricated by cutting a sheet of aluminum foil into a desired size and shape, and thus it may be suitable for shape adaptation by cutting. However, Quasi-WPT has a fundamental challenge: the return pass may be unstable since it is constructed by the stray capacitance between the RX and the source, which is highly affected by the external dielectric materials and the state of the TX sheet.

Based on the above review, we chose to design our system based on MCR-WPT due to two advantages of 1) robustness for misalignment between RX/TX, and 2) small interference from surrounding dielectric materials. Furthermore, we regard the simplicity of the system architecture as important since complicated architecture increases cost and requires a rich control system; this leads to difficulty in extension to larger WPT sheets, which is another reason we decided to use this method.

\section{DESIGN AND IMPLEMENTATION}

Given the above discussions, the core design criteria for our cuttable WPT sheet should be as follows:

1. The sheet should remain functional even after cutting manipulation.

2. The power supply area should be dense (i.e., power should be available from anywhere within the surface).

3. The system architecture should be applicable to large surfaces.

This goal brings about three main challenges, each related to one criterion. The first challenge is that typical circuits are not compatible with cutting. Thus, both the wiring topology and circuit design should be designed so that the remaining partition of the coil array works even after the sheet is partially cut. However, simple star or tree topology [33] is not suitable for TX coil arrays, as described in §3.2. Our solution is to use H-tree wiring which enables cutting TX coil arrays. Secondly, the dense power supply area requires the dense arrangement of the TX coils, although this leads to the performance degradation stemming from the inductive interference between adjacent TX coils, as illustrated in §3.3. Our solution is to employ time division power supply which 


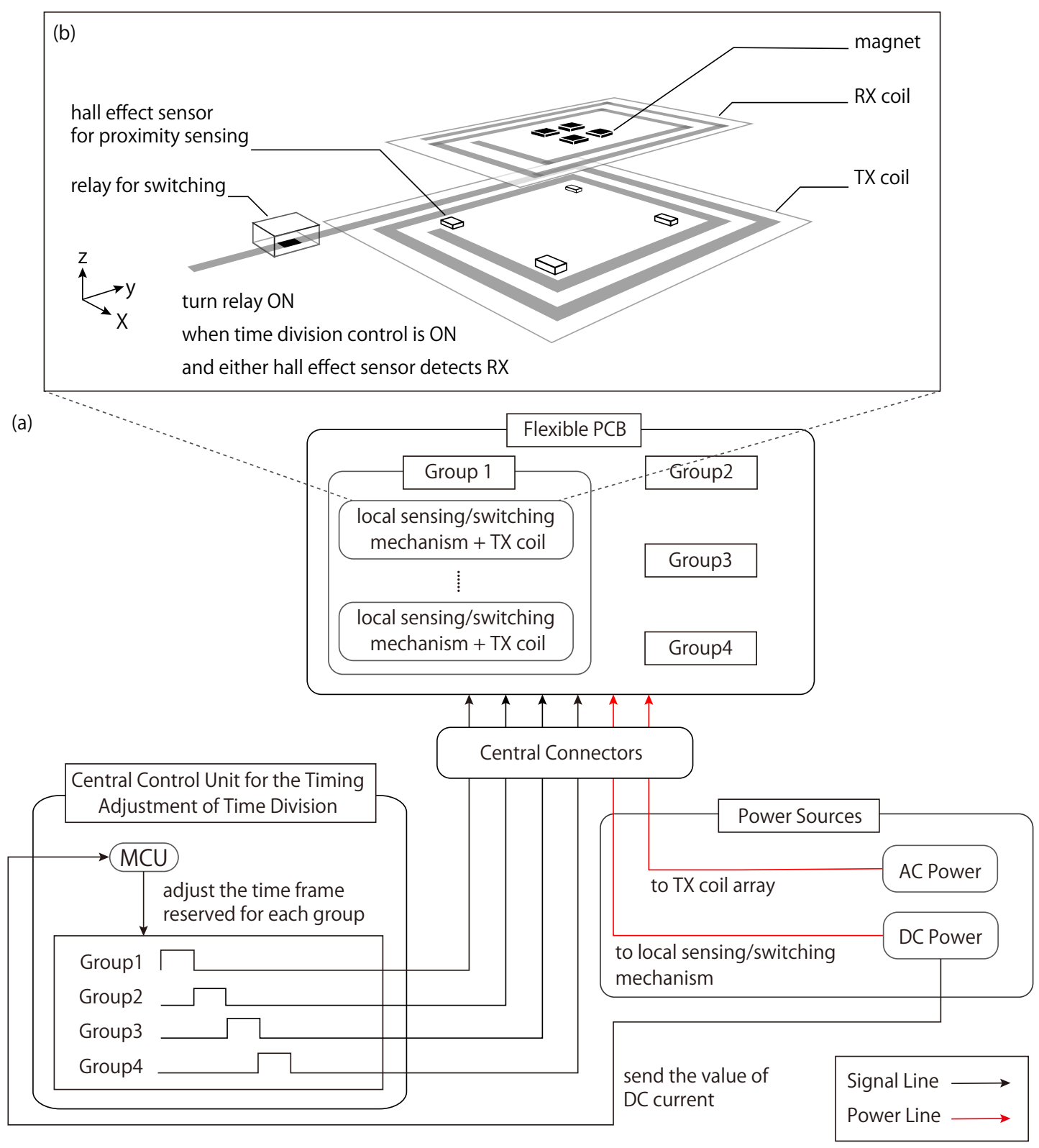

Fig. 2. System overview of cuttable WPT sheet. (a) Our system consists of three components: 1) a central control unit for the timing adjustment of time division, 2) a flexible PCB (TX coil array on the front side and local sensing/switching mechanism attached to each TX coil on the back side), and 3) AC and DC power sources. The flexible PCB is driven by AC/DC power sources and control signals for time division power supply through central connectors. (b) Each TX coil is activated when a proximity sensor attached to the TX detects RX and the time division signal for the TX is ON. 
avoids the simultaneous activation of adjacent TX coils. Lastly, it is difficult to guarantee scalability if we use the naive idea of central control; this tends to be complicated as the number of TX coils increases. Our solution is to partially offload the sensing/control procedure into local hardware embedded in each TX (detailed in §3.4) and minimize the role of central control unit (i.e, the central unit only provides power and sends out time division control signals).

In the remainder of this section, we first provide an overview of our system design, which combines the aforementioned three technologies. Then, we discuss the design of each core technology in detail. Lastly, we illustrate the implementation of our prototype.

\subsection{System Overview}

We show an overview of our system in Fig. 2. As shown in Fig. 2(a), our system consists of three main units: 1) a central control unit for the timing adjustment of time division operation, 2) a flexible PCB compatible with cutting (a TX coil array is on the front side, whereas the local sensing/switching mechanism is attached to each TX coil on the back side) and 3) AC and DC power sources; an AC power source (6.78 MHz) is used for WPT, whereas a DC power source supplies energy to the local sensing/switching mechanism. The power sources and control lines for driving both TX coil arrays and local sensing/switching module are connected via two input connectors, which are placed at the center of the flexible PCB; the only procedure necessary to make the system functional is to cut and paste the flexible sheet and plug the cables into these two connectors.

Next, we explain the procedure of power supply in this architecture. The objective of this procedure is to enhance energy efficiency and power capability by (i) avoiding the simultaneous activation of adjacent TX coils and (ii) selectively activating the TX coil closest to the RX coil. The brief description of this procedure begins with the role of the central unit. To avoid the simultaneous activation of adjacent TX coils, the central unit divides the TX coils into $N$ groups ( $N=4$ in the case of square TX coil arrays), each of which consists of non-adjacent TX coils connected in parallel, and transmits an activation signal to each group in turn. After the central unit, the next part is the local hardware module offloaded to each TX coil, which mainly consists of proximity sensing and switching mechanism. To activate the TX coil closest to the RX coil, the parallel connected TX coils are normally-OFF and the turning ON of the TX coil is triggered by the time division signal and proximity sensing unit; when a RX coil supplied with magnets approaches a TX unit, the hall effect sensors embedded in the TX detect the RX and turn the mechanical relay ON while the time division signal for the TX coil is ON, as shown in Fig. 2(b). Through this procedure, energy is only transmitted from TX coils with nearby RX coils, while avoiding the simultaneous activation of adjacent TX coils.

Finally, we note that the power transfer efficiency using a functional TX coil is nearly the same before/after cutting the sheet. Even after cut out into a random shape, the central time division control still avoids powering adjacent TX coils simultaneously and local proximity-sensing/switching mechanism activates TX coils closest to the RX coils and turns off the other TX coils. Thus, as long as the two aforementioned systems work correctly, the power transfer efficiency using the remaining TX coils doesn't get affected.

\subsection{H-Tree Wiring}

Employing H-tree wiring is our core idea to keep as many remaining TX coils to be functional as possible when the outer edge of the sheet is cut. This wiring is a fractal topology and is widely used in the field of LSI to equalize the distance from the center to each unit; this is beneficial for synchronizing the clock signal delivered to each endpoint [10]. In terms of our application, $\mathrm{H}$-tree wiring has three benefits: 1) the length of each feeder line becomes equal; this is important for suppressing the variation of electromagnetic properties between each feeder line, 2) the patterns extend from the center to the edges, which consequently enables the wire patterns on the 


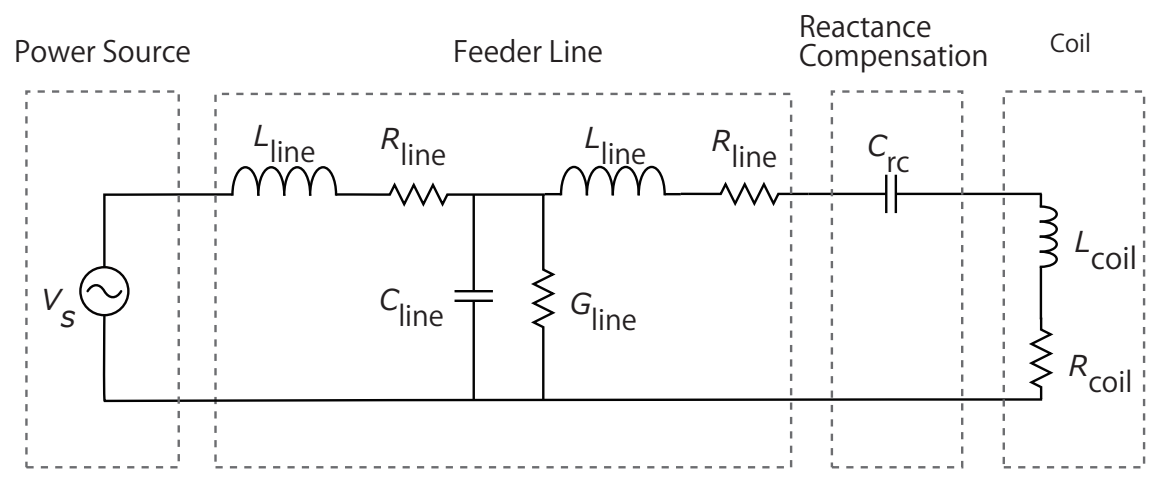

Fig. 3. The equivalent circuit of a TX coil seen from the central power source. This model consists of four parts; power source $\left(V_{s}\right)$, feeder line $\left(L_{\text {line }}, C_{\text {line }}, R_{\text {line }}, G_{\text {line }}\right)$, reactance compensation element $\left(C_{\mathrm{rc}}\right)$, and coil $\left(L_{\text {coil }}, R_{\text {coil }}\right) . R_{\text {coil }}$ is the parasitic resistance of coil. The value of $L_{\text {line }}, C_{\text {line }}, R_{\text {line }}$, and $G_{\text {line }}$ depends on the geometry of feeder line. Consequently, when the lengths of the feeder lines differ between each TX coil, the input impedance of each TX coil seen from the central power source varies intensely due to the effect of transmission line. Furthermore, we place a capacitor for reactance compensation before each TX coil. This element is necessary for taking a resonance in a TX coil at the operating frequency.

(a)

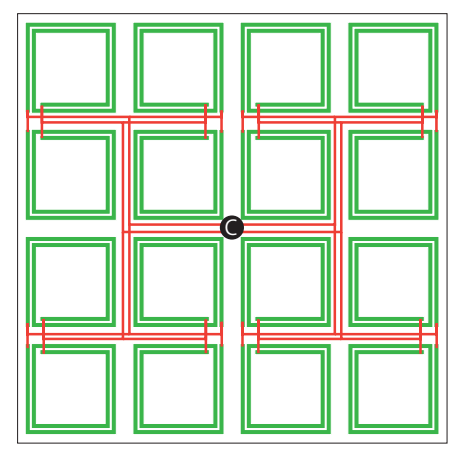

Functional Coil Dysfunctional Coil _ Power Line (b)
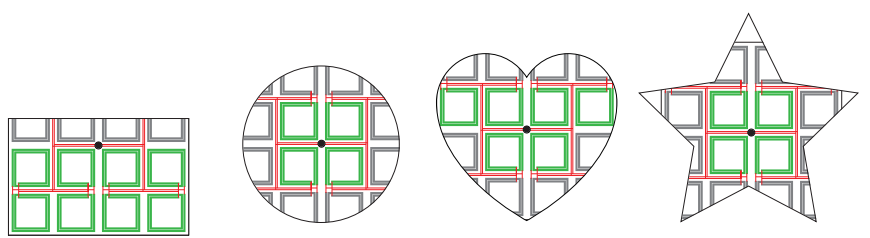

(c)
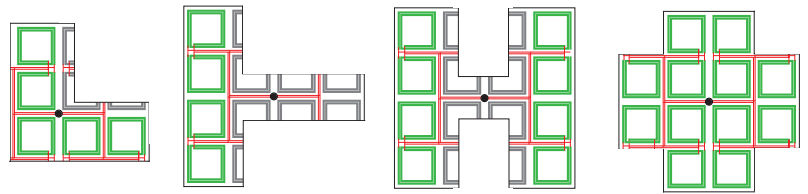

(d)
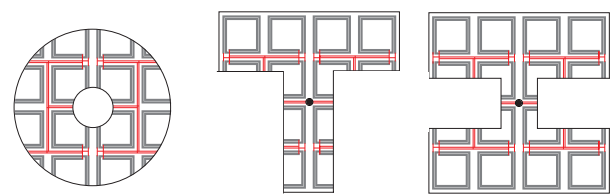

Fig. 4. (a) H-tree wiring (red) for a square TX coil array. This can support shapes such as (b) convex shapes (rectangle, ellipse, heart, and star) and (c) concave shapes (L, T, H, and +), (d) but cannot support shape-modification such as cutting off the input connectors or cutting the most fundamental branches. 


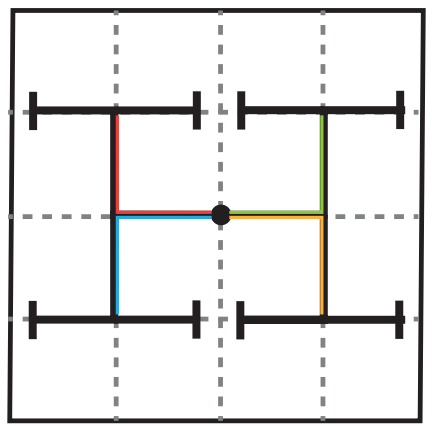

Fig. 5. To communicate the wiring layout with users, the front side features fine printed lines. Black bold lines express $\mathrm{H}$-tree wiring and four colored lines (red, green, yellow, and blue) mean the most fundamental wiring of H-tree wiring. Gray dotted grid lines represent the arrangement of TX coil arrays.

edges to become minor branches, 3) the fractal property makes it possible to expand the wiring pattern according to the number of elements in a straightforward manner.

Other candidates of wiring topology are simple star or tree wiring topology, each of which is used in the cuttable multi-touch sensor sheet [33]. Although these topologies have the benefits (2) and (3) of the H-tree, neither topology has the benefit (1). Therefore, the length of the feeder lines differs between each TX coil in the case of these wiring topologies. Consequently, the input impedance of each TX coil seen from the central power source varies intensely due to the effect of transmission line, as illustrated in Fig. 3; considering that resonant WPT systems require the precise adjustment of reactance (i.e., reactance compensation that cancels the imaginary part of input impedance at the operating frequency), this is cumbersome.

Due to benefit (2), the square TX coil arrays connected through H-tree wiring shown in Fig. 4(a) support a wide variety of shape adaptation. Fig. 4(b) and Fig. 4(c) show a set of supported cut-out shapes: convex shapes (rectangle, circle, heart, and star) and concave shapes ( $\mathrm{L}, \mathrm{T}, \mathrm{H}$, and +), respectively, can be made without losing the function of the sheet. However, as illustrated in Fig. 4(d), we must mention that there are some limitations for cutting square TX coil arrays connected to H-tree topology. First, a partially cut TX coil loses its function. This limitation becomes more apparent when the intended shape of the surface is convex shape except rectangle. Second, there are non-available cutting manipulations such as cutting off the input connectors (donut) or cutting the most fundamental branches (rotated 90 degree $\mathrm{T}$ and $\mathrm{H}$ ). The first limitation degrades the WPT coverage at the edge of cut-out shapes. The second limitation requires us to pay attention to both the placement of connectors and the pattern of $\mathrm{H}$-tree wiring. To alleviate these limitations, we introduce the guide paper pasted on the cuttable WPT sheet, as shown in Fig. 5. The guide paper mainly includes the layout of TX coil arrays (gray dotted grid line), H-tree wiring (black bold line), and most fundamental lines (red, green, yellow, and blue lines). As a rule of thumb, the cut-out shape will work as long as the fundamental lines and the central connectors remain on the guide paper.

Finally, we discuss the scalability (benefit (3)). Theoretically, H-tree topology supports to draw wiring of $2^{\mathrm{N}} \times 2^{\mathrm{N}}(N \geqq 1)$ square TX coil arrays without the shortage of the space of wiring due to the nature of fractal figure. However, there is a trade-off between the length of wiring from central connectors to each TX coil and the efficiency of the entire system; the longer wiring causes the larger energy loss. The in-depth investigation of the trade-off here requires us to improve the fabrication process of flexible substrates and optimize the size of TX coil, as described in $\S 6.1$. 
(a)

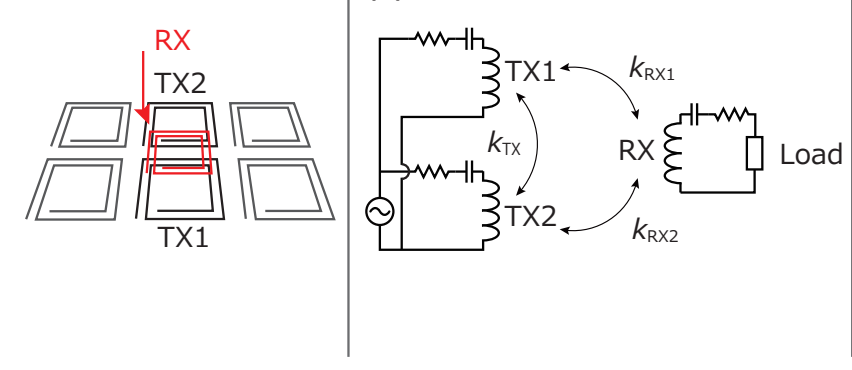

(c)

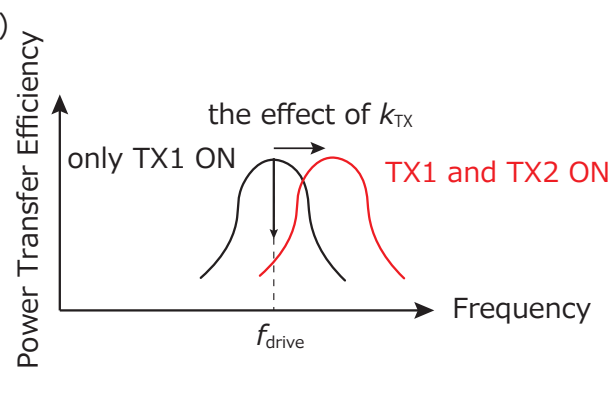

Fig. 6. A reduction in power transfer efficiency due to the magnetic coupling between adjacent TX coils. (a) Power transfer from multiple TX coils to a single RX coil (red). (b) The RX couples with each TX with a coupling coefficient of $k_{\mathrm{RX} 1}$ and $k_{\mathrm{RX} 2}$. TXs also couple with each other with a coupling coefficient of $k_{\mathrm{TX}}$. The two TXs are simultaneously driven in phase by the same voltage source. (c) Power transfer efficiency decreases because peak efficiency frequency is shifted due to the magnetic coupling of adjacent TXs $\left(k_{\mathrm{TX}}>0\right)[6]$.

\subsection{Time Division Power Supply}

In our time division scheme, the TX coils are grouped into several groups; each group consists of non-adjacent TX coils and the time division unit selects the group which power is delivered to. This way, as long as power is transmitted to each group in turn, adjacent TX coils never get simultaneously activated.

To help understand the necessity of time division power supply, we first explain the problem that occurs when adjacent TX coils operate simultaneously. Fig. 6(a) shows a primitive case; the RX coil is placed between two TX coils (TX1, TX2). Such situations occur frequently when multiple TX coils are densely arranged. In this case that both TX1 and TX2 are activated (i.e., no time division case), the equivalent circuit becomes as depicted in Fig. 6(b). In this circuit, the couplings between the coils are expressed by magnetic coupling coefficients, $k_{\mathrm{TX}}, k_{\mathrm{RX} 1}$, and $k_{\mathrm{RX} 2}$; this condition is called "cross-coupling". Some may think that in this situation, the RX receives more power compared to a single TX case since there are two TXs. However, in reality, it is known that the existence of $k_{\mathrm{TX}}$ detunes the resonant frequency of the system as shown in Fig. 6(c). Thus, the power transfer capability at the driving frequency $f_{\text {drive }}$ gets degraded [6,24].

A power supply mechanism should either avoid or compensate for the inductive interference between adjacent TX coils since this causes a shift in the system's optimal operating frequency and input impedance. There are two approaches to solve this: (a) dynamic compensation for the interference by using adaptive impedance tuning networks $[34,47]$ and (b) designing the system architecture so that adjacent TX coils do not get activated simultaneously $[4,38]$. The former pattern (a) requires much more rich hardware at each TX coil for the detection of interference and the compensating mechanism; this is not suitable for our intension, so we extend on the (b) case. As an example of the (b) case, Qi's TX array selectively activates only the closest TX coil to the RX coil; since it can select non-adjacent TX coils, it is obvious that significant interference does not occur. The key of this system is the position detection of the RX, where the central unit exhaustively searches for the TX which the RX is placed on [4]. The TX coils are activated in turn based on time division multiplexing [29, 52]. While this operation can avoid the magnetic interference between adjacent TX coils, this lacks scalability since the time for scanning all TX coils and the computational load for TX selection increase as the number of TX coils increases.

In contrast, our time division power supply scales well independent of the number of square TX coils and also avoids the cross-coupling of adjacent TX coils. Since the role of the central control unit is only to select the group 
(a)

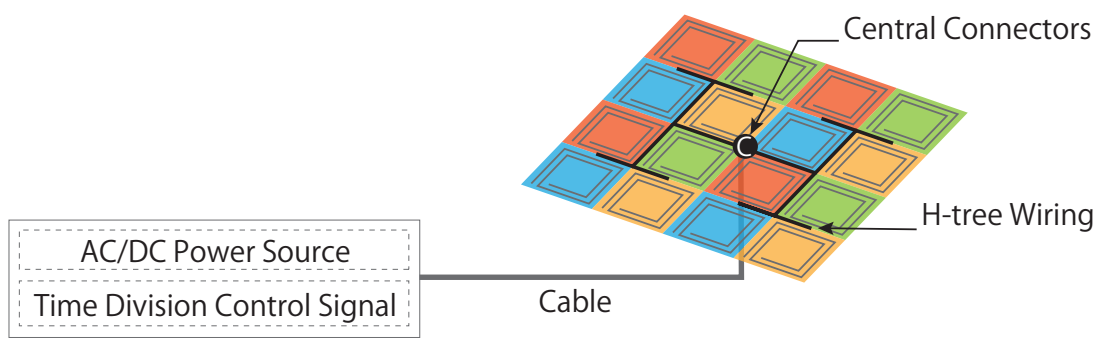

(b)
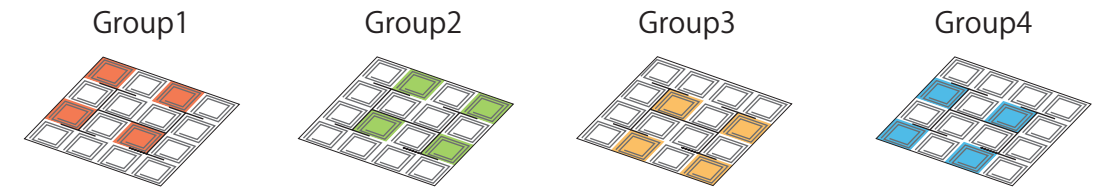

Group1

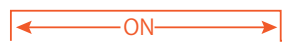

Group2

Group3

Group4
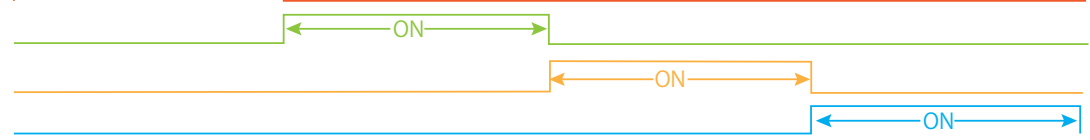

Fig. 7. The overview of time division power supply. (a) A square coil array is divided into four groups and connected with $\mathrm{H}$-tree topology. (b) We transmit power to each group in turn.
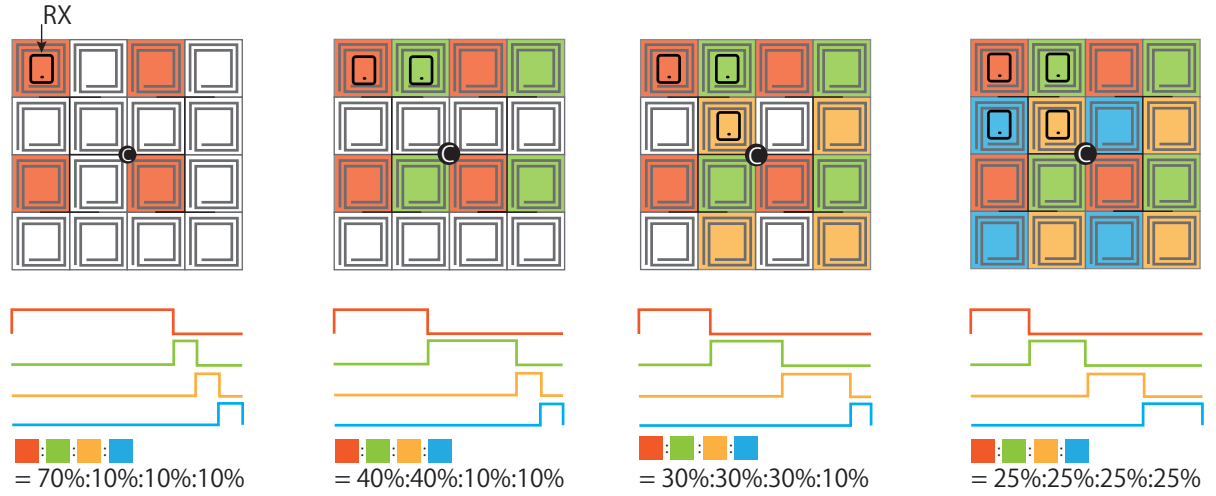

Fig. 8. Some examples of dynamic adjustment of the time frame reserved each group. The proportion of time frame is tuned by the number of the activating group. We can detect whether each group has RXs by monitoring the current consumption of the local proximity sensing and switching mechanisms. 
to which power is delivered, the number of necessary control channels is always $N$ (the number of groups), no matter what the number of the TX coils is.

The next question should be how many groups $(N)$ to compose. Since the amount of power that can be transferred to a TX coil becomes $1 / N$ of the total power capability in naive implementations, the number of groups should be minimized. Although we explained that the inductive interference between TX coils placed in proximity leads to negative impact on system performance, this negative impact is negligible when the distance between the TX coils is more than approximately a diameter of the coil. Fig. 7 shows time division power supply applied to square coil arrays. Based on the above observation, we divide the TX coils into four groups, which is the minimum number of division in square arrays, and connect them with $\mathrm{H}$-tree wiring in a straightforward manner.

Consideration should also be given to the control signals for time division power supply. To enhance the maximum power that can be supplied, we introduce dynamic adjustment of the time frames reserved for each group; in this way, the time reserved for the groups where no RX is placed is minimized. By monitoring the current consumption of the local proximity sensing and switching mechanisms, as described in §3.4, we can detect whether RXs are placed on the group or not; based on this information, we can adjust the time frame reserved for each group.

Fig. 8 shows some examples of time frame adjustment. In the case where no RX is placed on the array (i.e., inactive state) or an RX is placed on every group, the proportion of the assigned power supply time is $25 \%: 25 \%$ : $25 \%: 25 \%$. In the conditions that some groups have RXs placed on them and the other groups do not, the ratio of reserved power supply time is adjusted to $70 \%: 10 \%: 10 \%: 10 \%$ (only one group has one or more RXs on it), $40 \%: 40 \%: 10 \%: 10 \%$ (each of two groups has one or more RXs on it), or $30 \%: 30 \%: 30 \%: 10 \%$ (each of three groups has one or more RXs on it). While the time frame reserved for groups which no RXs are placed on should be zero, dynamic adjustment requires non-zero time period in such groups since the signal transmission of our system is one-way channel from the central control unit to the local sensing/switching module. Thus, we note that the change of consumption current in sensing/switching module (i.e, the power consumption for driving a switching element) of each group is only a cue for detecting whether RXs are placed on the group or not. While wireless communication module (e.g., bluetooth, Wi-Fi, and so on) can compensate for return channel, this necessitates a cumbersome setup, the construction of the reliable network, and MCU. These complexity is not compatible with our concept that system architecture should be simple. Thus, we avoid the use of wireless communication modules in our study. Furthermore, the optimization of time frame adjustment for groups where no RXs are placed may be considered (e.g., assigning less than $10 \%$ or assigning the time frame once at several times), but this is out of the scope in our work and we will consider this in the future work.

\subsection{Proximity Sensing and Switching Mechanism}

As described above, we connect the TX coils in parallel and activate each group in turn based on time division control. However, this leads to a problem specific to our scheme; a large proportion of energy gets consumed at the unloaded TX coil when loaded and non-loaded TX coils co-exist in the activated group. This causes critical energy loss in the system. To address this challenge, we present a hardware-based local proximity sensing and switching mechanism and install it into each TX coil. By introducing this key mechanism, we can selectively transfer power only when RX coils are in proximity, without employing any extra central control procedure. As a result, we can control the energy flow and achieve high-efficiency. The next question should be how we practically implement this conceptual mechanism.

As for the candidates for proximity sensors, representative examples are wireless communication modules (e.g., RFID [23], bluetooth, and Wi-Fi), hall effect sensors, detection coils [4], and optical (infrared) sensors. While each proximity sensor has different pros and cons, the proximity sensor in our system should be simple since 


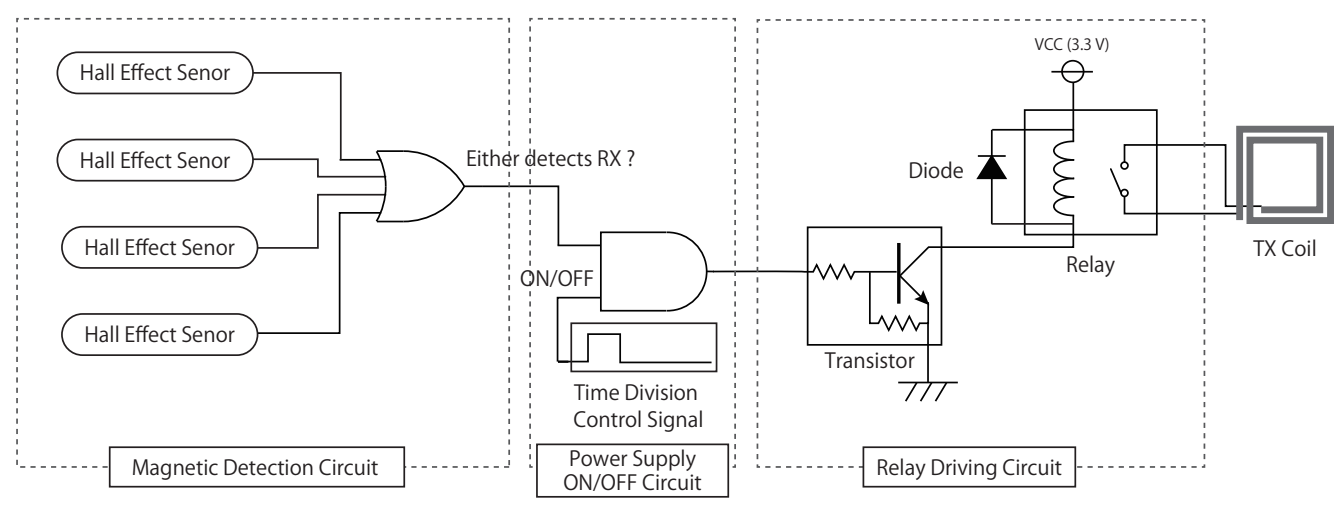

Fig. 9. Schematic diagram of local proximity sensing and switching mechanism. The relay in a TX is turned on only when the hall effect sensor detects an RX and a time division control signal for the TX is ON.

our proximity sensing and switching mechanism needs to conclude in each TX coil. Based on this consideration, the requirements of the proximity sensor are that 1) it is relatively simple, cheap, and does not require MCUs and 2) its detection threshold can be easily adjusted. The candidates for this are the proximity sensors other than wireless communication modules. Here, we chose hall effect sensors since the detection threshold can be easily adjusted by either circuitry or the geometry of the magnet. We noticed that the main disadvantage of hall effect sensor is that the external magnetic fields may interfere with the output value. Thus, we assume that our sheet cannot be applied to magnetized materials, although it should not be a problem for typical non-magnetic materials used in everyday items (e.g., wooden or plastic furniture, clothing, and bag).

Next, for switching components in high-frequency power circuits, the candidates are practically guaranteed to be mechanical relays and solid-state relays. The desirable characteristics in selecting the switching element are high OFF impedance, low ON impedance, and high-voltage/current tolerance. The former two are necessary to achieve high system efficiency, although these are not easy requirements to fulfill since 1) parasitic capacitance decreases impedance in high-frequencies (i.e., the current can not be stopped in OFF states) and 2) High-Q coils with low parasitic resistance are premised in resonant WPT systems and a few hundred $\mathrm{m} \Omega$ order losses can easily degrade system efficiency. The last one is necessary since resonant WPT systems easily induce highvoltage/current depending on the operation. Under these considerations, we decide that mechanical relays are appropriate since solid state devices suffer from the trade-off of voltage tolerance, high ON resistance, and junction capacitance, which makes it difficult to maintain the above-mentioned requirements.

\subsection{Implementation of Prototype}

To demonstrate the validity of our design, we implemented a functional prototype, as shown in Fig. 10 . The size of the prototype is $400 \mathrm{~mm} \times 400 \mathrm{~mm}$, the weight is $82 \mathrm{~g}$, and the sheet thickness is approximately $100 \mu \mathrm{m}$ without components, whereas, $5.7 \mathrm{~mm}$ considering the components. On the front side, a $4 \times 4$ TX coil array wired by H-tree topology is implemented. This coil array is connected to the AC power source through the central connector. The TX coils are $90 \mathrm{~mm} \times 90 \mathrm{~mm}$ 2-turn square coils with line width and spacing of $5 \mathrm{~mm}$ and $1 \mathrm{~mm}$. The thickness of the copper layer is $35 \mu \mathrm{m}$. On the back side, the proximity sensing and switching mechanism is installed into each TX coil. Both the control line for time division control and the DC power line are drawn according to H-tree topology. 
Front Side

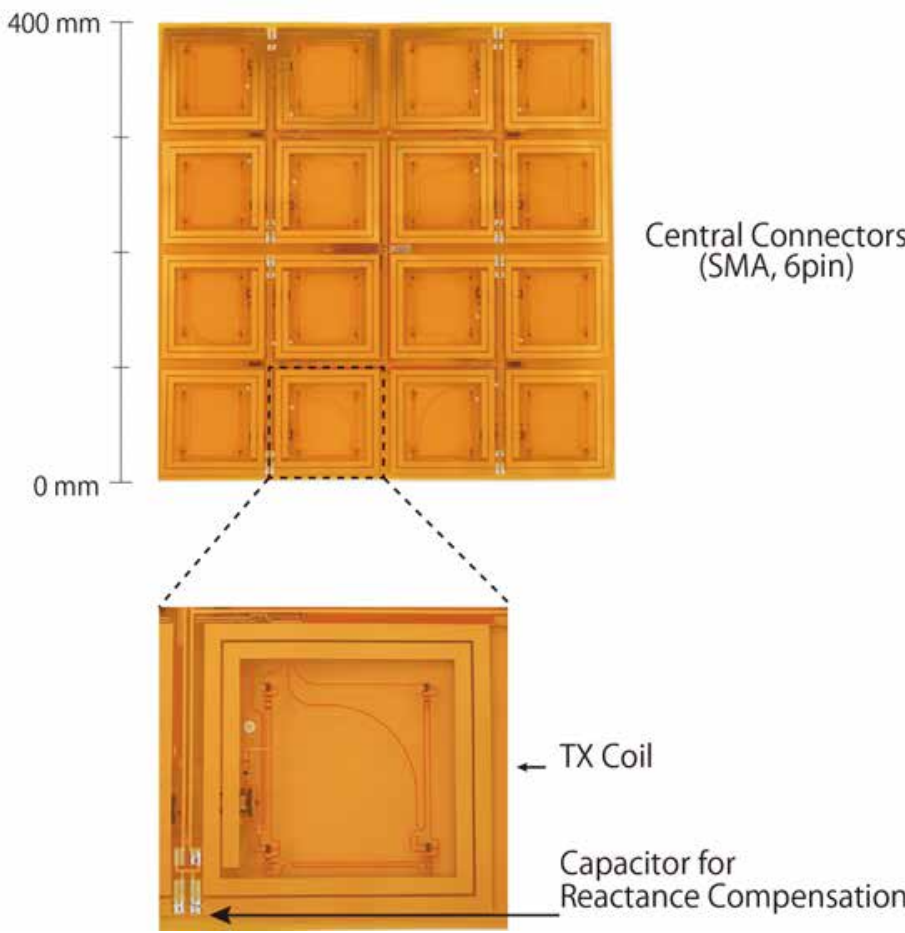

Back Side

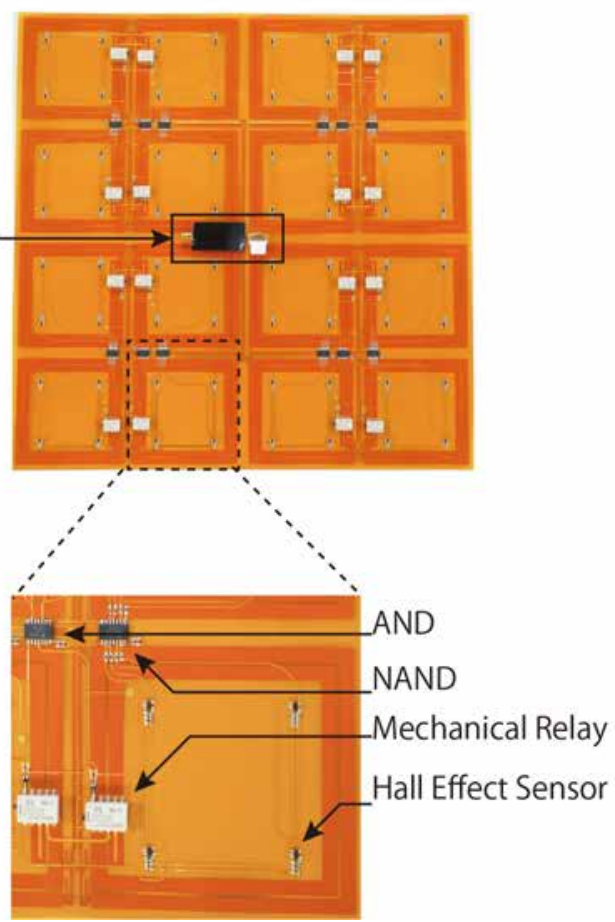

Fig. 10. Our prototype of cuttable wireless power transfer sheet for $6.78 \mathrm{MHz}$ band.

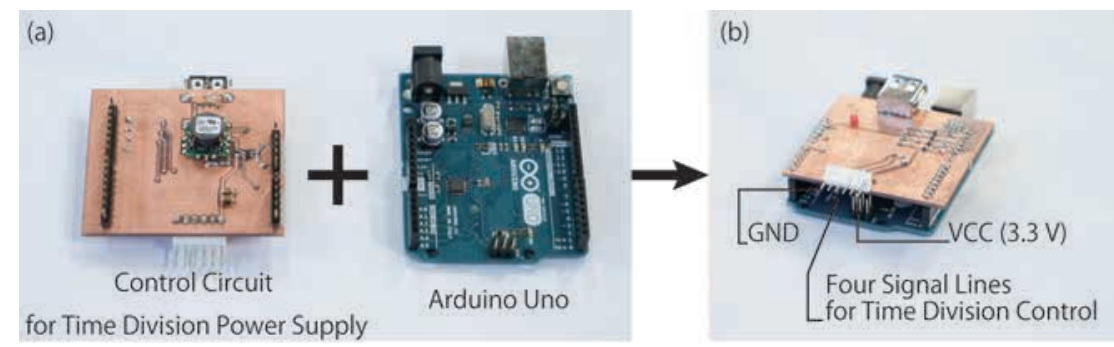

Fig. 11. Our prototype of control circuit for time division power supply. (a) This is implemented mainly by Arduino Uno and MOSFET. (b) Arduino Uno drives the control circuit for time division power supply.

The proximity sensing and switching mechanism consists of four hall effect sensors (BUS52025G-TR), a NAND circuit (TC74HC20AF(F)), an AND circuit (TC74RX08F(F)), a MOSFET (DTC123JETL), a diode (1SS355TE-17), and a mechanical relay (TQ2SA-3V-Z), which are connected as shown in Fig. 9. The reason for using TQ2SA-3V-Z as mechanical relays is that it has an ON impedance of $61 \mathrm{~m} \Omega$ and an OFF impedance of $42 \mathrm{k} \Omega$ at $6.78 \mathrm{MHz}$, and the maximum $\mathrm{AC}$ voltage/current tolerance of $125 \mathrm{~V} / 0.5 \mathrm{~A}$. These values fully meet three requirements as switching element described in §3.4. Also, the reason for using NAND circuits instead of OR circuits is that the hall effect 
sensor (BUS 52025G-TR) outputs $0 \mathrm{~V}$ when it detects magnetic flux and $3.3 \mathrm{~V}$ when there is no ambient magnetic field; the outputs of the hall effect sensors needs to be inverted. At the center of the sheet, two input connectors are mounted: an SMA connector for AC power supply and a 6-pin connector for DC power supply and time division control signals. As for the AC power source, we use off-the-shelf $6.78 \mathrm{MHz}$ class-D amplifiers (EPC 9065), which typically operate in voltage mode (i.e., the source impedance is low). The DC power is supplied by a $3.3 \mathrm{~V}$ voltage source.

As for driving frequency, we use $6.78 \mathrm{MHz}$ which is higher than the frequency used in Qi (i.e., around a hundred $\mathrm{kHz}$ ). The reason why we employ the higher frequency is that a TX coil with high Q-factor can be made thin and lightweight since the high inductance is not required and the skin depth is small [22, 28]. To explain this claim, we need to note that the figure of merit of WPT efficiency is the product of coupling factor $k$ and Q-factor $Q=\omega_{0} L / R$ [32]; this means that if Q-factor is large, the WPT efficiency remains high even if coupling $k$ is small (i.e., it achieves high-efficiency in misaligned conditions). Since the frequency used in Qi is approximately $100 \mathrm{kHz}$ and the frequency we use is $6.78 \mathrm{MHz}$, coils with magnitudes of higher inductance are required to achieve high Q-factor with Qi specifications; the problem here is that high-inductance coils require more turns which makes coils bulky. Moreover, since the skin depth of conductors is inverse proportional to the square root of frequency, high-frequency coils an be designed on thin conductors without a significant increase in loss; in the low-efficiency case such as Qi, the performance significantly degrades when deployed with thin conductors.

The control circuit for time division power supply is implemented by using MOSFETs and Arduino Uno, as shown in Fig. 11. This circuit also provides the 3.3 V voltage and therefore has 6 outputs: four time division control signals lines, a DC power line, and a GND line. The minimum period of time division scheme in our prototype is $40 \mathrm{~ms}(=10 \mathrm{~ms} \times 4)$ considering the opening and closing time of the relay ( $4 \mathrm{~ms}$ and $4 \mathrm{~ms}$, respectively) and thus, we set up the period of time division power supply as $400 \mathrm{~ms}$, taking a 10 -fold safety factor.

\section{APPLICATIONS}

Here, we present some potential applications for our cuttable wireless power transfer sheet. The thin and flat properties of the sheet enable seamless integration with board and paper; the nature of flexibility and lightness as well as the above-mentioned properties also bring about good compatibility with cloth. Furthermore, due to its subtractive fabrication process, our cuttable wireless power transfer sheet enables the users to quickly explore the combinations of shape and fabricate a charger that works on surfaces of everyday objects. To provide a proof of concept for this, we made four applications; wireless charging furniture, bag, jacket, and craft.

\subsection{Wireless Charging Furniture}

Wireless charging pads for mobile electronic devices are commercially available in recent years [1,2]. Some companies like IKEA provide built-in wireless charger kits that can be integrated with wooden or plastic furniture such as side tables and shelves. In contrast, we present wireless charging furniture based on subtractive fabrication. Fig. 12 shows an example of a cut-and-pasting wireless charger built into furniture to blend in the surroundings more naturally. At the back side of the wooden tray, our cuttable WPT sheet is attached. The sheet successfully detected a smartphone and a reading lamp on the tray and provided enough power $(2.5 \mathrm{~W})$ for both of the devices. Different from the prototypes of the existing 2-D wireless power charger research [30,38], our proposed sheet is highly customizable and naturally integrated with users' favorite furniture.

\subsection{Wireless Charging Bag and Jacket}

Many people carry smartphones and other electric devices in the bags or pockets on the clothes. This is most likely the most appropriate moment to charge mobile devices because they are not in use at this time. Fig. 13 and Fig. 14 show use case examples of making a wireless in-bag or in-jacket charger. A mobile lithium-ion battery 

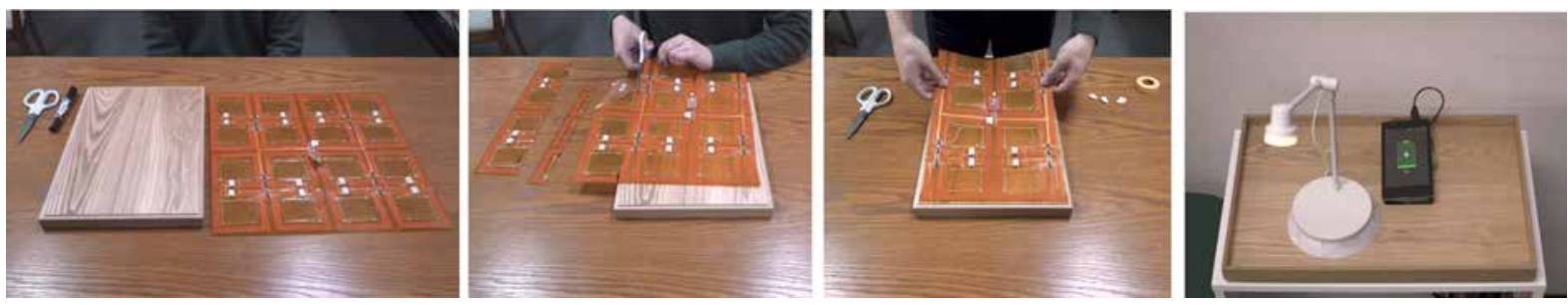

Fig. 12. Wireless charging furniture.
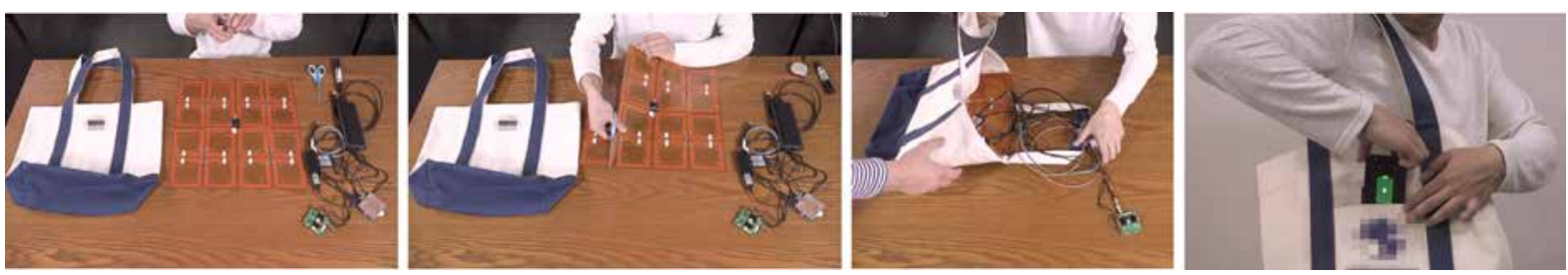

Fig. 13. Wireless charging bag.
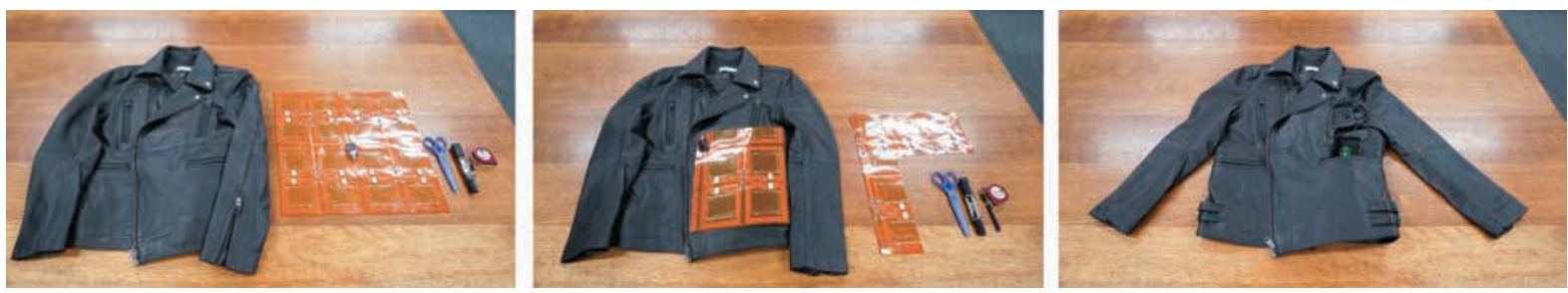

Fig. 14. Wireless charging jacket.
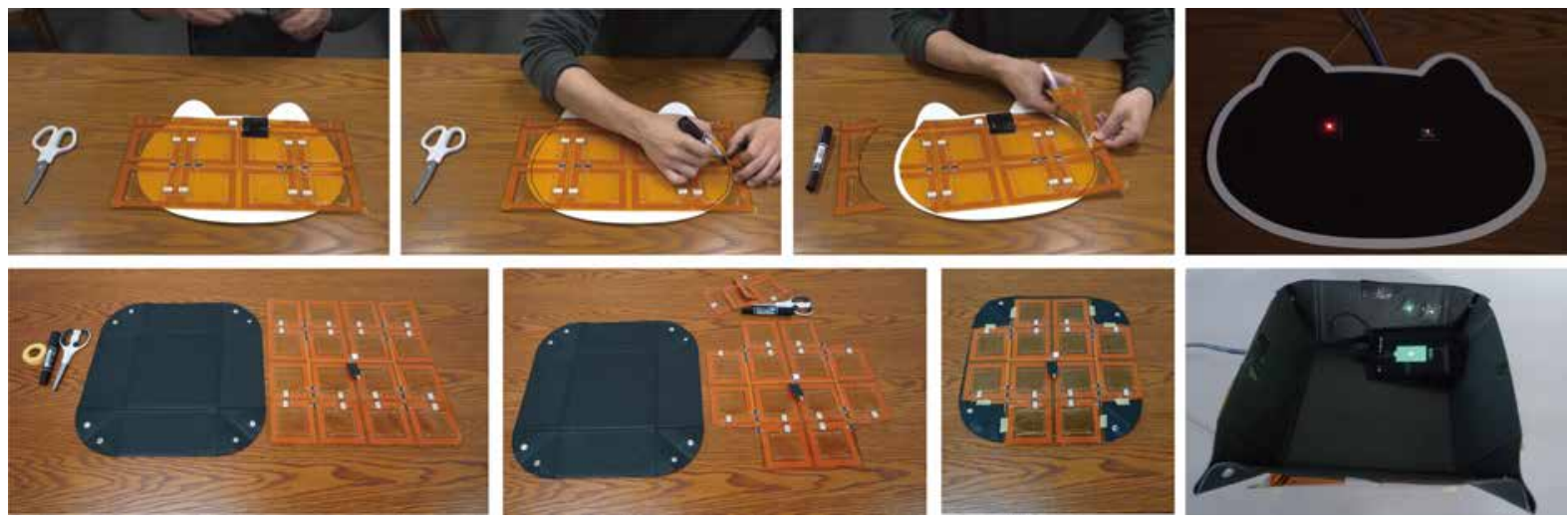

Fig. 15. Wireless charging craft. 
pack is used to feed power to the cuttable sheet. Different from a Qi-based wireless charger [3], the elasticity of the sheet does not ruin the flexibility of the bag or jacket and the sheet covers a large wireless charging area.

\subsection{Wireless Charging Craft}

As our sheet is a prototyping tool, it enables non-experts to transform hand-made crafts into a wireless charger. For example, children can easily learn to build WPT surfaces through prototyping. Artists also may quickly prototype story-telling artworks. Fig. 15 shows two examples of wireless charging craft: a hand-crafted cat-shaped wireless charging mat and a leather-crafted wireless charging box. It took only a few minutes to make the mat and box compatible with wireless charging. The mat and box support wireless charging of multiple devices while the flexibility is unimpaired. By using the mat, we lit up two wirelessly powered LEDs to express the flashing eye of the cat. The mobile box can be used to charge a smartphone in the box and light up the wirelessly powered LEDs. Compared to simply arranging wireless charger units [1,2], our design process requires neither a bulky apparatus $[34,47]$ nor an additional control unit for controlling the current flow of the entire TX coil array [38] to solve the magnetic interference between each unit.

\section{EVALUATIONS}

Here, we conducted a series of evaluations to confirm the requirements of the cuttable WPT sheet. First, we evaluated its cuttability (i.e., the area of the WPT sheet which remains functional after it is partially cut) through simulation. Then, we confirmed that the proximity sensing mechanism can detect receivers placed at any position on the WPT sheet. Finally, we investigated the power transfer efficiency through measurements in practical configurations (e.g., co-located with external objects, RX misaligned, etc.).

\subsection{Cuttability Simulation}

First, we evaluated the cuttability of our cuttable WPT sheet in a similar way as cuttable multi-touch sensor [33]. We implemented a simulation framework, which takes a set of 2-D shapes as input. Then, we simulated an $800 \mathrm{~mm} \times 800 \mathrm{~mm}$ cuttable WPT sheet covered with a square array of $8 \times 8 \mathrm{TX}$ coils. The geometry of TX coil is the same as the coil of our prototype and the space between the coils is $10 \mathrm{~mm}$. We selected the following cut-out shapes for simulation of cuttability: rectangle and ellipse. Rectangle and ellipse are basic shapes commonly used in a wide variety of objects. In [33], the parameters of cut-out shapes is the position, scale, and rotation. In contrast, we fixed the rotation of cut-out shapes since $\mathrm{H}$-tree is not robust for the rotation of cut-out shapes; rotation, other than at 0 degree, causes the more intersection with the wiring. We set up the minimum size of a bounding box of the cut-out shapes as $594 \mathrm{~mm} \times 594 \mathrm{~mm}$. For each cut-out shape, 15 patterns of non-uniform scales and 100 patterns of random locations (a minimum step size of $10 \mathrm{~mm}$ ) are randomly generated. The simulation calculates the performance index as similar to [33] for each combination of scale $\times$ location of the cut-out shape . We note that the cut-out shapes that cover out of the sheet or do not contain the central connectors are not considered. The cuttability of rectangle and ellipse in our cuttable WPT sheet is $58.9 \% \pm 11.3 \%$ and $45.9 \% \pm 7.0 \%$, respectively. While these value lower for cuttable multi-touch sensor, which also considers the rotation of cut-out shapes (96\%), this is reasonable since partially cut coils lose function, whereas electrodes do not.

To enhance the cuttability, overlaying (i.e., stacking) of two or more different topologies such as X-tree topology [7] will be a solution since it is likely that at least one wiring remains functional with more redundancy. In addition to adding a different topology, overlaying two same layouts rotated by 90 degrees will also enhance the tolerance to the rotation of cut-out shapes. In the future, we will perform the above-mentioned methods and increase redundancy by stacking multiple wiring topologies using a multi-layer flexible PCB. 
(a)
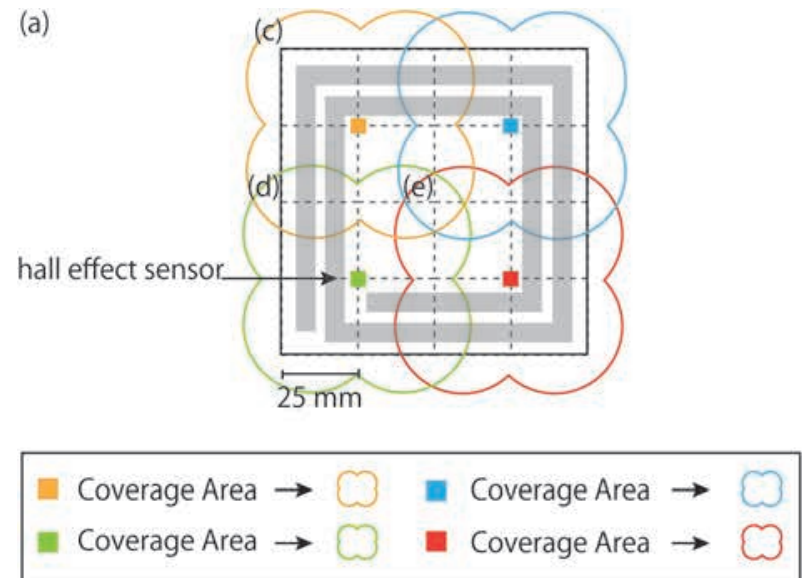

(b)
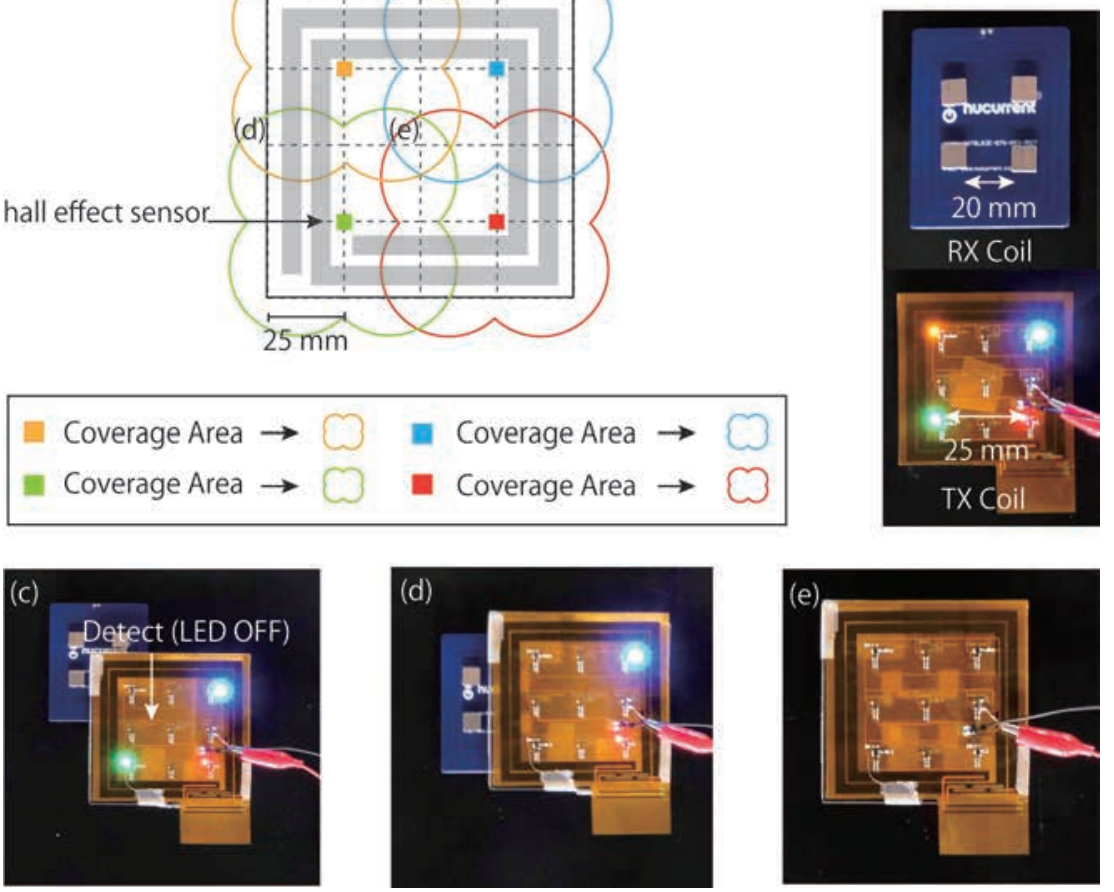

Fig. 16. Detection area of four hall effect sensors on a TX coil. (b) We estimated the detection area and (c)(d)(e) checked its estimation against measurement roughly. We measured by watching the ON/OFF of the LED which reflects the output of the nearby hall effect sensor.

\subsection{Detection Area of Hall Effect Sensor}

Next, we evaluated the coverage area of the hall effect sensors to make sure that our prototype can detect RXs placed anywhere on the sheet. To meet this requirements, we installed four hall effect sensors per one TX coil on $2 \times 2$ grid points with an interval of $25 \mathrm{~mm}$, as shown in Fig. 2(b). On the RX coil, we installed four $10 \mathrm{~mm} \times 10 \mathrm{~mm}$ neodymium magnets on $2 \times 2$ grid points with an interval of $20 \mathrm{~mm}$; this is also shown in Fig. 2(b). This configuration is based on the maximum distance that a hall effect sensor can detect; a case where the TX coil and RX coil are separated with a distance of $2 \mathrm{~mm}$ (in the direction of $z$-axis) was considered. This distance assumes the thickness of everyday cover cloth or plywood. Under this condition, the maximum detection distance is about $7 \mathrm{~mm}$ (this represents distance on the $x-y$ plane). Based on this consideration, we placed four neodymium magnets on $2 \times 2$ at $20 \mathrm{~mm}$ interval, as shown in Fig. 16(b). Next, we estimated the coverage area over which each hall effect sensor can detect a RX, as depicted in Fig. 16(a). Then, we checked if the proximity sensing mechanism works as intended by performing simple tests employing indicator LEDs; the RX was placed on several lattice points and the LED turned off when the associated hall effect sensor detected the RX in proximity (See Fig. 16(a) and Fig. 16(b)). Based on this setup, we checked the output of hall effect sensor, as illustrated in Fig. 16(c)(d)(e). Fig. 16 shows that at least one of the four hall effect sensors of each TX coil can detect a RX coil as long as it is placed on top of a TX coil. Considering the TX coil array is a periodic, this means that our proximity sensing mechanism fully covers our prototype. 


\subsection{Evaluation of Power Transfer Efficiency}

Here, we evaluated the power transfer efficiency of our system through measurements in the following configurations: (a) time division control activated (proposed method) and deactivated (baseline method), (b) interfering materials existing in proximity, (c) the distance between the TX/RX coil varied, and (d) the TX coils bent to a various degrees.

To calculate the power transfer efficiency from §5.3.1 to §5.3.4, we used the formulation of Zargham et al. that derives the upper limit of power transfer efficiency (i.e., the efficiency with perfect impedance matching) from the scattering parameters (S-parameters) [51], considering that various maximum efficiency point tracking methods are studied in the literature [27]. Lastly, we investigated the total DC-to-DC power transfer efficiency that our system can achieve in $\S 5.3 .5$; note that the optimization of the AC-DC/DC-AC conversion is not the intention of this study and this is just to give a reference for future system design. This total power transfer efficiency is calculated as the proportion of DC power delivered to the load within the output power of the DC power source.

In the measurements, we used custom-designed RX boards which consists of a coil, a full-bridge rectifier, and a $5 \mathrm{~V}$ output buck-converter. The coil is a $80 \mathrm{~mm} \times 80 \mathrm{~mm}, 6$ turn square coil; the width of the coil pattern is $1.25 \mathrm{~mm}$ and the spacing is $0.5 \mathrm{~mm}$.

5.3.1 Activating/Deactivating Time Division Control. To confirm the concept of time division power supply, we compared the power transfer efficiency with the time division schema activated (proposed method) and deactivated (baseline). We measured the scattering parameters (S-parameters) from the central connector (SMA) to the RX coil using a vector network analyzer (VNA), as shown in Fig. 17(a). We performed this measurement at the extracted lattice points on the TX coil array illustrated in Fig. 17(b) and calculated the efficiency using the obtained S-parameters. Considering the symmetry of the TX coil, we selected the six positions, as shown in Fig. 17(b), to fully cover the area of the TX coil. For this measurement, the control circuit for time division control outputs are configured as following: (i) activating one group and deactivating the others, which corresponds to our proposed schema, and (ii) activating all groups, which corresponds to the situation which time division control is not applied. Fig. 17(c) and (d) show the plots of power transfer efficiency when time division is activated and deactivated. It can be seen that by activating the time division control, the average efficiency on the grid points improves from $35 \%$ to $65 \%$ and the minimum efficiency increases from $2 \%$ to $24 \%$.

5.3.2 External Materials in Proximity. Next, we consider the situations in which external materials exist in proximity to our WPT sheet; there are two questions we address here: (i) do the substances which cover or are embedded into this WPT sheet affect system performance? and (ii) how do foreign substances placed between the TX and RX affect system performance? To examine this issue, we measured the power transfer efficiency with materials placed on the back of the WPT sheet and between the WPT sheet and RX, as shown in Fig. 18; the external substances we considered here are wood, cloth, acrylic, and metal. We fixed the thickness of each external material to $2 \mathrm{~mm}$. Fig. 19(a) shows the measured power transfer efficiency with external substances placed between the WPT sheet and RX, whereas Fig. 19(b) presents the measured power transfer efficiency with external substances placed on the back of the WPT sheet. From these results, we can see that only metal significantly affects the power transfer efficiency and the others have almost no effect on the system. It can be assumed that this degradation in the metal case occurs since magnetic flux causes eddy current and consequently, cannot penetrate metal. Therefore, we considered that for the case that the metal is placed on the back of the WPT sheet, this degradation can be mitigated by placing three pieces of ferrite sheet (TDK IFL04-200NB300X200) between the external metal and WPT sheet; ferrite sheets can form a path for the magnetic flux to flow along, which mitigates the metallic substance to some degree [50]. From these evaluations, we confirmed that our sheet is compatible with non-metal materials; we also showed that even in the metal case, the interference can be mitigated in some cases using ferrite sheets. 
(a)

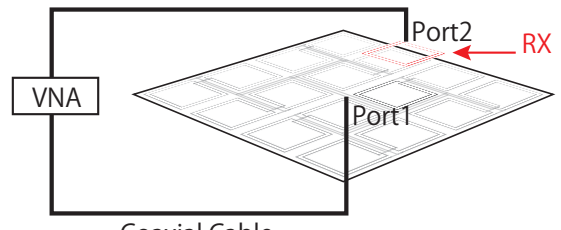

Coaxial Cable

(b)

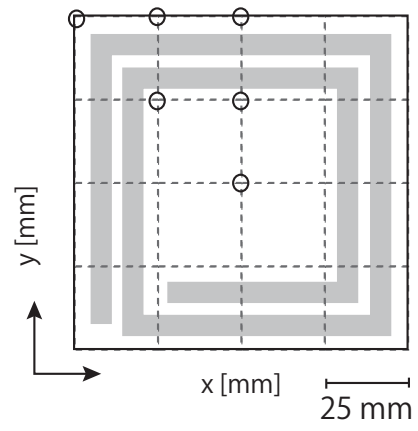

(c)

power transfer efficiency

with time division power supply

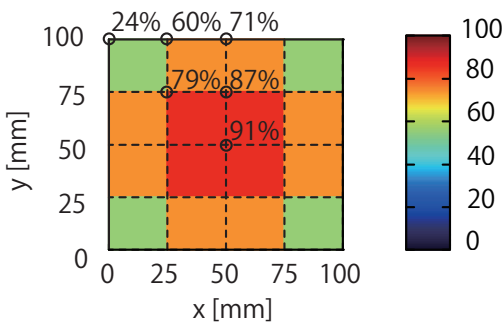

Ave. : 65\%, Min : 24\%, Max : 91\%

(d)

power transfer efficiency without time division power supply

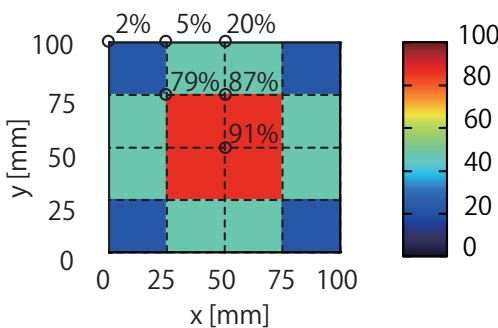

Ave. : 35\%, Min : 2\%, Max : 91\%

Fig. 17. Power transfer efficiency with and without time division control. (a) We measured the S-parameters between the central connector (Port1) and the load port on the RX coil (Port2) using a VNA. (b) We calculated the maximum available AC to AC power transfer efficiency at each lattice point (o) based on the obtained S-parameters. (c) The plot of power transfer efficiency with time division power supply. (d) The plot of power transfer efficiency without time division power supply.

5.3.3 Variation of Distance. The power transfer efficiency with the TX to RX distance is frequently used as a figure of merit in MCR-WPT studies. Generally, MCR-WPT can realize high-efficiency over mid-range (i.e., approximately the diameter of the coil) [16]. To test power transfer efficiency with distance variance, we used the same experimental setup as shown in Fig. 17(a) and placed an RX coil at the center of a TX coil. Then, we measured power transfer efficiency with the distance between the coils swept from $0 \mathrm{~mm}$ to $70 \mathrm{~mm}$ with a step of $10 \mathrm{~mm}$. Fig. 20 shows the experiment results. These results show that we can obtain high efficiency (over 70\%) as long as the distance is smaller than $40 \mathrm{~mm}$ (i.e., approximately half of the diameter of the TX coil). It is noted that the main usage scenario of this system is to charge RXs placed close to the WPT sheet and therefore, optimization for extending range is not our main interest; this efficiency is likely to be enhanced by further optimization of coil patterns and advanced flexible coil design methods [22, 28].

5.3.4 Transmitter Coils Being Bent. Our prototype sheet is flexible and consequently, it can be applied to curved and flexible surfaces. However, it can be assumed that the curvature of the TX coil changes the system characteristics; this section addresses this concern by measuring the WPT link under the situations that the TX coil is bent. In the measurements, we configured the TX coil and the RX coil as shown in Fig. 21(a). We conducted 


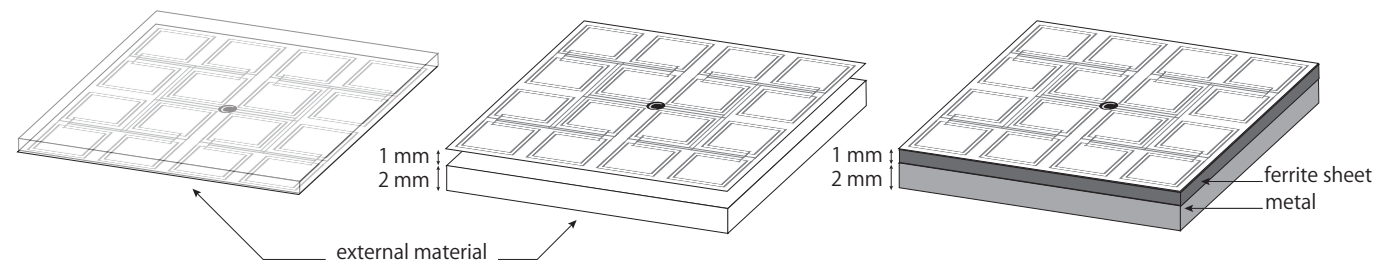

Fig. 18. The measurement setup. We placed external materials (wood, cloth, acrylic, and metal) (a) between RX and our WPT sheet and (b) on the back of our WPT sheet. When the material is metal, we also covered ferrite sheet between metal and WPT sheet.

(a)

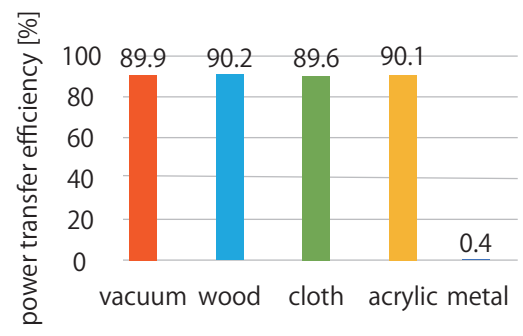

(b)

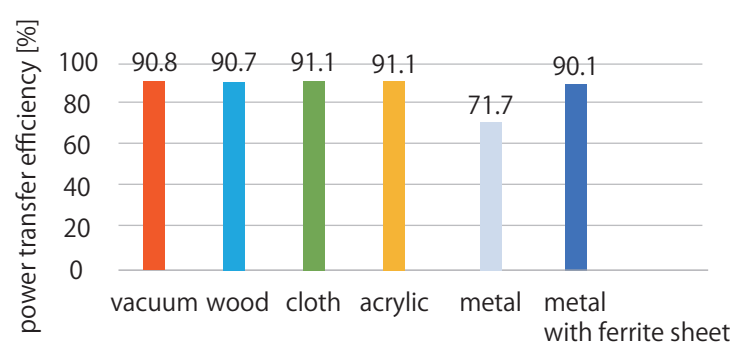

Fig. 19. Power transfer efficiency with materials (wood, cloth, acrylic, and metal) placed (a) between the WPT sheet and RX and (a) on the back of the WPT sheet. Metal degrades power transfer efficiency, but for the case that the metal is placed on the back of the WPT sheet, this degradation can be mitigated by placing three pieces of ferrite sheet (TDK IFL04-200NB300X200) between the external metal and WPT sheet.

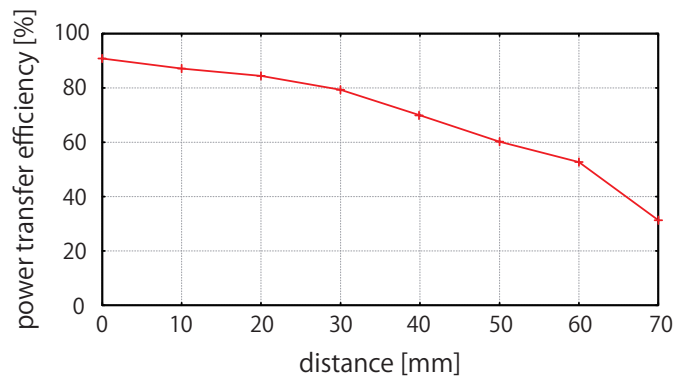

Fig. 20. Power transfer efficiency for distance. We changed the distance between an RX coil and an TX coil in our prototype with a step of $10 \mathrm{~mm}$ and calculated maximum power transfer efficiency at each distance.

measurements with seven bending radii which range from $40 \mathrm{~mm}$ to $140 \mathrm{~mm}$ and evaluated the obtainable power 
(a)

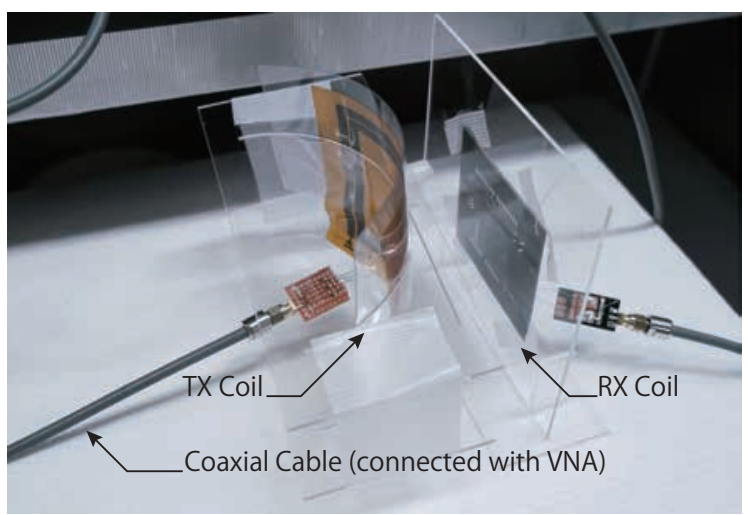

(top view)

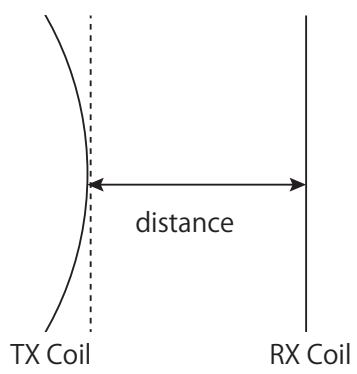

(b)

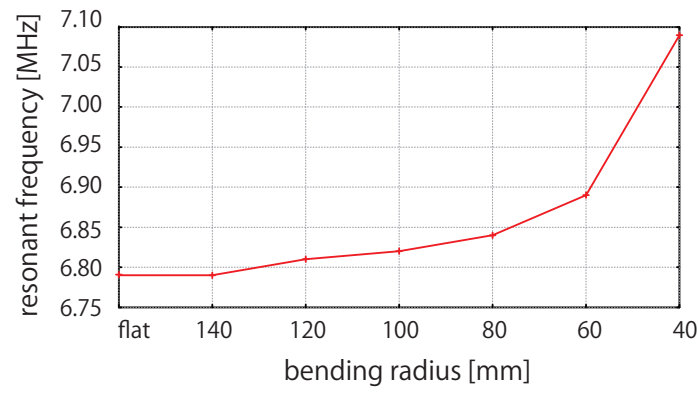

(c)

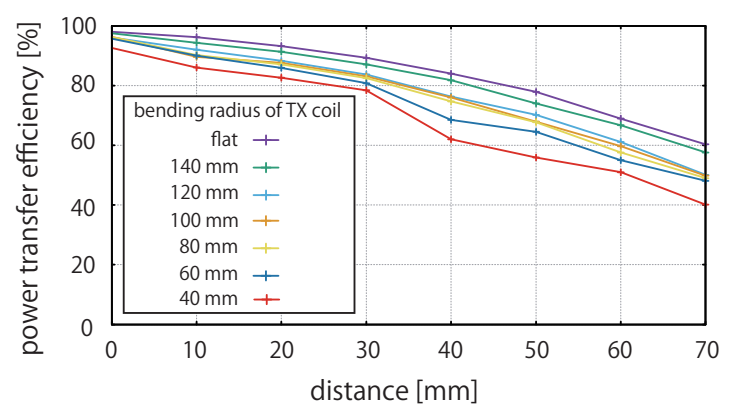

Fig. 21. (a) Experimental setup for calculating resonant frequency and power transfer efficiency. (b) Resonant frequency for bending radius. (c) Power transfer efficiency as a function of distance for TX coil bending.

transfer efficiency and the resonant frequency of the TX; we also compared these results with the "flat" case where there is no bending.

Fig. 21(b) shows the variation of the resonant frequency of the TX as bending radius is varied. The resonant frequency increases with curvature; this result can be interpreted that the inductance of the coil decreases as the coil gets bent further since the resonant frequency can be formulated as $f_{0}=1 / 2 \pi \sqrt{L C}$. Fig. 21(c) is the plot of power transfer efficiency as a function of distance, each line of which represents the radius of the TX coil bending. It can be seen that the efficiency drops as the curve becomes sharper. This efficiency drop is attributed to the drop of inductance as well as the reduction of the coupling coefficient between the TX and RX coil; the drop of coupling coefficient occurs since the flux that interlinks with the TX/RX coil decreases.

5.3.5 DC-to-DC Efficiency. Finally, we investigated the total power transfer efficiency from the DC power source to the load (i.e. smartphone); the DC power source here refers to the power source that feeds the AC power source. Despite our cuttable wireless power transfer sheet is a proof-of-concept system and the optimization of the AC-to-DC, DC-to-AC, and power conditioning stages are out of scope for this study, we show its end-to-end efficiency for future reference. We used a smartphone as the destination of power delivery since smartphones are frequently used in our daily life among mobile devices. We connected the charging port of the smartphone to the custom designed RX board, which receives power from the TX and provides a voltage regulated output. 
As a representative point, we placed the RX at the center of a TX coil used in our prototype. The RX board was configured so that it supplies $5 \mathrm{~V}, 1 \mathrm{~A}$ to the smartphone via charging port. The measured power transfer efficiency under this setup was $60 \%$ when a smartphone was charged. At this time, the measured power supplied to the smartphone was $4.2 \mathrm{~W}(=4.7 \mathrm{~V} \times 0.9 \mathrm{~A})$, whereas the measured output power of the DC power source was $7.0 \mathrm{~W}(=14 \mathrm{~V} \times 0.5 \mathrm{~A}$ ). This result shows the efficiency dropped from $91 \%$ (AC-to-AC efficiency) to $60 \%$ (DCto-DC efficiency). The causes of this drop are not just the neglected loss of the AC power source, rectifier, and buck-converter, but also due to the non-optimized operation point of the buck-converter; this occurs since the input-impedance of a buck-converter is a function of the input voltage. Therefore, additional control mechanisms to dynamically track the optimal operation point are necessary; fortunately, countermeasures to this problem is presented in former literature such as [27] and these methods can be applied to our system in the future.

\section{LIMITATIONS AND FUTURE WORKS}

Besides the limitation described above, there are other technical limitations to the cuttability and usability of our system.

\subsection{Cuttability}

One main limitation is the existence of non-cuttable components on the proposed sheet. In our prototype, we used rigid COTS components, which form non-cuttable areas. Users can easily avoid these components when cutting; therefore, this does not significantly degrade the delivered experience and is not an urgent issue; although, for further optimization, the use of flexible organic components is a promising option to fabricate a fully cuttable sheet $[18,19,48]$.

Our implementation requires the connector for the power sources to be located at the center of a sheet. Consequently, only one functional WPT surface can be cut out of a sheet. This is not a big problem when the size of the original sheet and the cut out sheet are similar, although as the original sheet gets larger, the cut off portion of the sheet gets inevitable; this cut off portion is wasted and degrades cost-efficiency. To alleviate this problem, we are investigating the idea of multiple spatially distributed power supply points on a single sheet; this enables several WPT surfaces to be cut out from one sheet.

The size of TX coil is also an important factor. While the bigger coil can transfer energy over a larger distance, this decreases resolution and therefore, cutting even a small portion of the coil results in a larger non-functional area. Consequently, there is a trade-off when selecting the coil size; smaller coil leads to the less damaged area but power transfer distance becomes smaller. Considering this trade-off, we plan to optimize coil size through electromagnetic field simulations.

As for this study, we assumed that the largest sheet size is $400 \mathrm{~mm} \times 400 \mathrm{~mm}$, which was the maximum size of flexible PCB available from the PCB manufacturing service we used. Thus, there is only $4 \times 4$ array about the configuration of square TX coil arrays considering the proper size of TX coil to charge smartphones. Assuming that we can access to B-to-B large-scale PCB fabrication services, which are based on roll-to-roll process [11], this restriction would not exist; some manufacturers can fabricate the PCBs as large as $1 \mathrm{~m}$ times a few hundred meters. Furthermore, printing technologies for functional electronic components are rapidly advancing; this is a promising technology for integrating the wiring and flexible organic components so that the fabrication process concludes in a single printing procedure [38].

\subsection{Usability}

Near the boundary of each TX coil, there are areas where significant drops in power transfer efficiency are observed; this occurs due to the magnetic flux canceling out, as shown in Fig. 17. Avoiding these efficiency drops is outside the scope of this study; therefore, we simply placed mobile devices so they avoid such areas in the 
WPT furniture demonstration and also needed alignment of coil position in the WPT bag/jacket demonstration. To address this issue, we will integrate RX arrays which are designed based on genetic algorithm to enhance the bottom of power transfer efficiency [36].

In our work, we only focused on square coil geometry. However, regular hexagonal or triangle coil are also suitable as the unit of a dense coil array. In our future works, we will investigate these shapes including the proper wiring topology, which has three features of $\mathrm{H}$-tree.

Although the detection range is limited to $7 \mathrm{~mm}$ due to the limitation of the magnetic proximity sensor, it successfully detects devices that are placed directly onto the power transfer sheet. In the case that some applications require detection of loads placed at larger distances, other detection mechanisms may be used [4, 23].

Finally, we plan to conduct user studies to further understand the usability of our system. The participants would compare two fabrication processes for custom-designed wireless powered surfaces: 1) cutting and pasting our WPT sheet based on the interaction that our prototype enables and 2) manually arranging and interconnecting WPT units as in a conventional procedure such as Qi. For this user study, we will also further develop the minor details of our WPT sheet such as adding redundancy and optimizing TX coil size.

\section{CONCLUSION}

In this paper, we introduced a cuttable wireless power transfer sheet along with its critical technical principles: $\mathrm{H}$-tree wiring for cutting a sheet from the outside and time division power supply for avoiding magnetic coupling between TX coils. We provided concrete applications showing how we envision this sheet being used in everyday objects. To study the possibilities of our system, we presented the current implementing prototype and evaluated cuttability and power transfer efficiency in various configurations. In the future, we will enhance the cuttability and usability. Finally, we hope that this article will pave the way for WPT sheets and spur more research in ubiquitous WPT surfaces.

\section{ACKNOWLEDGMENTS}

This work was supported by JST ERATO, Japan under Grant No.: JPMJER1501. We would like to thank Koya Narumi for making a nice figure for our paper. We also thank Masaru Takagi for his implementation of Android application.

\section{REFERENCES}

[1] 2008. Wireless Power Consortium. Retrieved May 11, 2018 from https://www.wirelesspowerconsortium.com/

[2] 2015. AirFuel Alliance: Home. Retrieved May 11, 2018 from http://www.airfuel.org/

[3] 2017. LiveFree Backpack: Perfect 24/7 Bag with wireless charging - Kickstarter. Retrieved May 11, 2018 from https://www.kickstarter com/projects/961207811/livefree-backpack-perfect-24-7-bag-with-wireless-c

[4] 2017. The Qi Wireless Power Transfer System Power Class 0 Specification Parts 1 and 2: Interface Definitions. Technical Report. Wireless Power Consortium.

[5] Johnson I Agbinya. 2015. Wireless power transfer. River Publishers.

[6] D. Ahn and S. Hong. 2013. Effect of Coupling Between Multiple Transmitters or Multiple Receivers on Wireless Power Transfer. IEEE Transactions on Industrial Electronics 60, 7 (2013), 2602-2613. https://doi.org/10.1109/TIE.2012.2196902

[7] Halil B Bakoglu. 1990. Circuits, Interconnections, and Packaging for VLSI. (1990).

[8] J. Dai and D. C. Ludois. 2015. Biologically inspired coupling pixilation for position independence in capacitive power transfer surfaces. In 2015 IEEE Applied Power Electronics Conference and Exposition (APEC). 3276-3282. https://doi.org/10.1109/APEC.2015.7104822

[9] Artem Dementyev, Hsin-Liu (Cindy) Kao, and Joseph A. Paradiso. 2015. SensorTape: Modular and Programmable 3D-Aware Dense Sensor Network on a Tape. In Proceedings of the 28th Annual ACM Symposium on User Interface Software and Technology (UIST '15). 649-658. https://doi.org/10.1145/2807442.2807507

[10] A. L. Fisher and H. T. Kung. 1985. Synchronizing Large VLSI Processor Arrays. IEEE Trans. Comput. C-34, 8 (1985), 734-740. https: //doi.org/10.1109/TC.1985.1676619 
[11] N. W. Gong, C. Y. Wang, and J. A. Paradiso. 2012. Low-cost sensor tape for environmental sensing based on roll-to-roll manufacturing process. In 2012 IEEE Sensors. 1-4. https://doi.org/10.1109/ICSENS.2012.6411347

[12] Andrew W Green and JT Boys. 1994. $10 \mathrm{kHz}$ inductively coupled power transfer-concept and control. In IEEE Power Electronics and Variable-Speed Drives. 694-699. https://doi.org/10.1049/cp:19941049

[13] C. K. Harnett. 2017. Tobiko: A Contact Array for Self-Configuring, Surface-Powered Sensors. In Proceedings of the 2017 CHI Conference on Human Factors in Computing Systems (CHI '17). 2024-2028. https://doi.org/10.1145/3025453.3025504

[14] A. Hashizume, Y. Narusue, Y. Kawahara, and T. Asami. 2017. Receiver localization for a wireless power transfer system with a 2D relay resonator array. In 2017 IEEE International Conference on Computational Electromagnetics (ICCEM). 127-129. https://doi.org/10.1109/ COMPEM.2017.7912817

[15] David Holman and Roel Vertegaal. 2011. TactileTape: Low-cost Touch Sensing on Curved Surfaces. In Proceedings of the 24th Annual ACM Symposium Adjunct on User Interface Software and Technology (UIST '11 Adjunct). 17-18. https://doi.org/10.1145/2046396.2046406

[16] S. Y. R. Hui, W. Zhong, and C. K. Lee. 2014. A Critical Review of Recent Progress in Mid-Range Wireless Power Transfer. IEEE Transactions on Power Electronics 29, 9 (2014), 4500-4511. https://doi.org/10.1109/TPEL.2013.2249670

[17] F. Jolani, Y. q. Yu, and Z. Chen. 2015. A planar positioning-free magnetically-coupled resonant wireless power transfer. In 2015 IEEE Wireless Power Transfer Conference (WPTC). 1-3. https://doi.org/10.1109/WPT.2015.7140176

[18] Martin Kaltenbrunner, Matthew S White, Eric D Głowacki, Tsuyoshi Sekitani, Takao Someya, Niyazi Serdar Sariciftci, and Siegfried Bauer. 2012. Ultrathin and lightweight organic solar cells with high flexibility. Nature communications 3, 770 (2012). https://doi.org/10. 1038/ncomms 1772

[19] Kazunori Kuribara, He Wang, Naoya Uchiyama, Kenjiro Fukuda, Tomoyuki Yokota, Ute Zschieschang, Cherno Jaye, Daniel Fischer, Hagen Klauk, Tatsuya Yamamoto, et al. 2012. Organic transistors with high thermal stability for medical applications. Nature communications 3, 723 (2012). https://doi.org/10.1038/ncomms1721

[20] Andre Kurs, Aristeidis Karalis, Robert Moffatt, John D Joannopoulos, Peter Fisher, and Marin Soljačić. 2007. Wireless power transfer via strongly coupled magnetic resonances. Science 317, 5834 (2007), 83-86. https://doi.org/10.1126/science.1143254

[21] C. Liu, A. P. Hu, and N. K. C. Nair. 2011. Modelling and analysis of a capacitively coupled contactless power transfer system. IET Power Electronics 4, 7 (2011), 808-815. https://doi.org/10.1049/iet-pel.2010.0243

[22] I. Lope, C. Carretero, J. Acero, J. M. Burdio, and R. Alonso. 2013. Printed circuit board implementation of small inductors for domestic induction heating applications using a planar litz wire structure. In 2013 Twenty-Eighth Annual IEEE Applied Power Electronics Conference and Exposition (APEC). 2402-2407. https://doi.org/10.1109/APEC.2013.6520632

[23] Nicolai Marquardt, Alex S. Taylor, Nicolas Villar, and Saul Greenberg. 2010. Rethinking RFID: Awareness and Control for Interaction with RFID Systems. In Proceedings of the SIGCHI Conference on Human Factors in Computing Systems (CHI '10). ACM, New York, NY, USA, 2307-2316. https://doi.org/10.1145/1753326.1753674

[24] K. Miwa, H. Mori, N. Kikuma, H. Hirayama, and K. Sakakibara. 2013. A consideration of designing coil array in wireless power transfer with magnetically coupled resonance. In 2013 Asia-Pacific Microwave Conference Proceedings (APMC). 1215-1217. https: //doi.org/10.1109/APMC.2013.6695075

[25] K. Mori, H. Lim, S. Iguchi, K. Ishida, M. Takamiya, and T. Sakurai. 2012. Positioning-Free Resonant Wireless Power Transmission Sheet With Staggered Repeater Coil Array (SRCA). IEEE Antennas and Wireless Propagation Letters 11 (2012), 1710-1713. https: //doi.org/10.1109/LAWP.2013.2239600

[26] Y. Narusue, Y. Kawahara, and T. Asami. 2013. Impedance matching method for any-hop straight wireless power transmission using magnetic resonance. In 2013 IEEE Radio and Wireless Symposium (RWS). 193-195. https://doi.org/10.1109/RWS.2013.6486685

[27] Yoshiaki Narusue, Yoshihiro Kawahara, and Tohru Asami. 2017. Maximizing the efficiency of wireless power transfer with a receiver-side switching voltage regulator. Wireless Power Transfer 4, 1 (2017), 42-54. https://doi.org/10.1017/wpt.2016.14

[28] Y. Nishizawa, Y. Narusue, and Y. Kawahara. 2018. Enhancing the quality factor of thin film printed coils for efficient wireless power transfer. In 2018 IEEE Radio and Wireless Symposium (RWS). 25-27. https://doi.org/10.1109/RWS.2018.8304936

[29] Aditya Shekhar Nittala, Anusha Withana, Narjes Pourjafarian, and Jürgen Steimle. 2018. Multi-Touch Skin: A Thin and Flexible Multi-Touch Sensor for On-Skin Input. In Proceedings of the 2018 CHI Conference on Human Factors in Computing Systems (CHI '18). ACM, New York, NY, USA, Article 33, 12 pages. https://doi.org/10.1145/3173574.3173607

[30] A. Noda and H. Shinoda. 2011. Selective Wireless Power Transmission Through High-Q Flat Waveguide-Ring Resonator on 2-D Waveguide Sheet. IEEE Transactions on Microwave Theory and Techniques 59, 8 (2011), 2158-2167. https://doi.org/10.1109/TMTT.2011. 2156425

[31] A. Noda and H. Shinoda. 2018. Antinull 2-D Waveguide Power Transfer Based on Standing Wave Diversity. IEEE Transactions on Microwave Theory and Techniques 66, 1 (2018), 306-318. https://doi.org/10.1109/TMTT.2017.2721403

[32] Takashi Ohira. 2014. Extended kQ product formulas for capacitive-and inductive-coupling wireless power transfer schemes. IEICE Electronics Express 11, 9 (2014), 1-7. https://doi.org/10.1587/elex.11.20140147

[33] Simon Olberding, Nan-Wei Gong, John Tiab, Joseph A. Paradiso, and Jürgen Steimle. 2013. A Cuttable Multi-touch Sensor. In Proceedings of the 26th Annual ACM Symposium on User Interface Software and Technology (UIST '13). 245-254. https://doi.org/10.1145/2501988.2502048

Proc. ACM Interact. Mob. Wearable Ubiquitous Technol., Vol. 2, No. 4, Article 190. Publication date: December 2018. 
[34] J. Park, Y. Tak, Y. Kim, Y. Kim, and S. Nam. 2011. Investigation of Adaptive Matching Methods for Near-Field Wireless Power Transfer. IEEE Transactions on Antennas and Propagation 59, 5 (2011), 1769-1773. https://doi.org/10.1109/TAP.2011.2123061

[35] A. K. Pickering, Richard Hull, J. E. Hawk, Arindam Phani, C. W. Van Neste, and Thomas Thundat. 2015. Quasi-wireless surface power and control for battery-free robotics. Wireless Power Transfer 2, 2 (2015), 134-142. https://doi.org/10.1017/wpt.2015.15

[36] T. Sasatani, Y. Narusue, Y. Kawahara, and T. Asami. 2016. Genetic algorithm-based design of receiving resonator arrays for wireless power transfer via magnetic resonant coupling. In 2016 IEEE Wireless Power Transfer Conference (WPTC). 1-4. https://doi.org/10.1109/ WPT.2016.7498842

[37] James Scott, Frank Hoffmann, Mike Addlesee, Glenford Mapp, and Andy Hopper. 2002. Networked Surfaces: A New Concept in Mobile Networking. Mob. Netw. Appl. 7, 5 (2002), 353-364. https://doi.org/10.1023/A:1016539632660

[38] Tsuyoshi Sekitani, Makoto Takamiya, Yoshiaki Noguchi, Shintaro Nakano, Yusaku Kato, Takayasu Sakurai, and Takao Someya. 2007. A large-area wireless power-transmission sheet using printed organic transistors and plastic MEMS switches. Nature materials 6, 6 (2007), 413-417. https://doi.org/10.1038/nmat1903

[39] Philipp Steurer and Mani B. Srivastava. 2003. System Design of Smart Table. In Proceedings of the First IEEE International Conference on Pervasive Computing and Communications (PERCOM '03). 473-480. https://doi.org/10.1109/PERCOM.2003.1192772

[40] C. J. Stevens. 2015. Magnetoinductive Waves and Wireless Power Transfer. IEEE Transactions on Power Electronics 30, 11 (2015), 6182-6190. https://doi.org/10.1109/TPEL.2014.2369811

[41] Y. G. Su, S. Y. Xie, A. P. Hu, C. S. Tang, W. Zhou, and L. Huang. 2017. Capacitive Power Transfer System With a Mixed-Resonant Topology for Constant-Current Multiple-Pickup Applications. IEEE Transactions on Power Electronics 32, 11 (2017), 8778-8786. https: //doi.org/10.1109/TPEL.2016.2640314

[42] Yuki Tajima, Akihito Noda, and Hiroyuki Shinoda. 2016. Signal and power transfer to actuators distributed on conductive fabric sheet for wearable tactile display. In International AsiaHaptics conference. Springer, 163-169. https://doi.org/10.1007/978-981-10-4157-0_28

[43] M. Takamiya, T. Sekitani, Y. Miyamoto, Y. Noguchi, H. Kawaguchi, T. Someya, and T. Sakurai. 2007. Design Solutions for a Multi-Object Wireless Power Transmission Sheet Based on Plastic Switches. In 2007 IEEE International Solid-State Circuits Conference. Digest of Technical Papers. 362-363. https://doi.org/10.1109/ISSCC.2007.373444

[44] Kristof Van Laerhoven, Nicolas Villar, Albrecht Schmidt, H-W Gellersen, Maria Hakansson, and Lars Erik Holmquist. 2003. Pin\&Play: the surface as network medium. IEEE Communications Magazine 41, 4 (2003), 90-95. https://doi.org/10.1109/MCOM.2003.1193980

[45] C.W. Van Neste, J.E. Hawk, Arindam Phani, J.A.J. Backs, Richard Hull, Tinu Abraham, S.J. Glassford, A.K. Pickering, and Thomas Thundat. 2014. Single-contact transmission for the quasi-wireless delivery of power over large surfaces. Wireless Power Transfer 1,2 (2014), 75-82. https://doi.org/10.1017/wpt.2014.9

[46] Nicolas Villar, Florian Block, Dave Molyneaux, and Hans Gellersen. 2006. VoodooIO. In ACM SIGGRAPH 2006 Emerging Technologies (SIGGRAPH '06). Article 36. https://doi.org/10.1145/1179133.1179170

[47] William H Von Novak, Charles E Wheatley III, Stanley S Toncich, and Ernest T Ozaki. 2012. Adaptive impedance tuning in wireless power transmission. US Patent 8,338,991.

[48] Matthew S White, Martin Kaltenbrunner, Eric D Głowacki, Kateryna Gutnichenko, Gerald Kettlgruber, Ingrid Graz, Safae Aazou, Christoph Ulbricht, Daniel AM Egbe, Matei C Miron, et al. 2013. Ultrathin, highly flexible and stretchable PLEDs. Nature Photonics 7, 10 (2013), 811-816. https://doi.org/10.1038/nphoton.2013.188

[49] Raphael Wimmer and Patrick Baudisch. 2011. Modular and Deformable Touch-sensitive Surfaces Based on Time Domain Reflectometry. In Proceedings of the 24th Annual ACM Symposium on User Interface Software and Technology (UIST '11). 517-526. https://doi.org/10. $1145 / 2047196.2047264$

[50] X. Xin, D. R. Jackson, and J. Chen. 2017. Wireless Power Transfer Along Oil Pipe Using Ferrite Materials. IEEE Transactions on Magnetics 53, 3 (2017), 1-5. https://doi.org/10.1109/TMAG.2016.2626254

[51] M. Zargham and P. G. Gulak. 2012. Maximum Achievable Efficiency in Near-Field Coupled Power-Transfer Systems. IEEE Transactions on Biomedical Circuits and Systems 6, 3 (2012), 228-245. https://doi.org/10.1109/TBCAS.2011.2174794

[52] Yang Zhang, Chouchang (Jack) Yang, Scott E. Hudson, Chris Harrison, and Alanson Sample. 2018. Wall++: Room-Scale Interactive and Context-Aware Sensing. In Proceedings of the 2018 CHI Conference on Human Factors in Computing Systems (CHI '18). ACM, New York, NY, USA, Article 273, 15 pages. https://doi.org/10.1145/3173574.3173847

Received May 2018; revised August 2018; accepted October 2018 